\title{
ReSidentielle MobILITÄT IM SPANNUNGSFELd ZWISCHEN VULNERABILITÄT UND STRUKTURELLER INTEGRATION - DETERMINANTEN DER WOHNPRÄFE- RENZEN UND BINNENMIGRATION VON GEFLÜCHTETEN AUS AFGHANISTAN IN ÖSTERREICH
}

\author{
Josef КонцваснеR, Institut für Stadt- und Regionalforschung, \\ Österreichische Akademie der Wissenschaften
}

\section{INHALT}

1 Einleitung.

2 Freiwillige versus erzwungene Binnenmobilität:

Motive und Auswirkungen von Dispersionspolitiken

3 Wissenschaftlicher State of the Art

4 Zur räumlichen Dispersion und Binnenmigration von Geflüchteten in Österreich 107

5 Empirische Resultate auf Basis der Interviews ................................................. 111

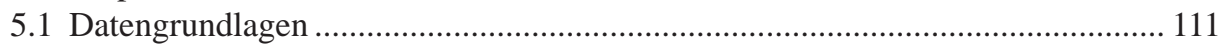

5.2 Räumlich-regionale Wohnpräferenzen und Binnenmigration.............................. 112

5.3 Die Attraktivität des Urbanen: Wien als Zuwanderungsmagnet in Österreich

5.4 Plädoyers für die Integration in ländlichen Gemeinden

5.5 Manifestationen der Vulnerabilität im ländlich-kleinstädtischen Raum und deren Auswirkungen auf Binnenmigrationsentscheidungen

5.6 Die Lokalisation der ethnischen Community als Determinante räumlicher Mobilitätsentscheidungen

5.7 Die Bedarfsorientierte Mindestsicherung (BMS) ein Kausalfaktor der Binnenmigration?

6 Conclusio

7 Bibliographie. 125 


\section{Einleitung}

Die sogenannte „refugee crisis“ von 2015/16 konfrontierte in Österreich alle größeren urbanen Zentren, Kleinstädte und sogar viele der kleineren Gemeinden mit der Herausforderung, binnen kurzer Zeit die Infrastruktur zu schaffen, erhebliche Zahlen an Geflüchteten unterzubringen und über einen längeren Zeitraum zu versorgen. Die meisten dieser Geflüchteten verfügten über keinerlei oder sehr geringe Kenntnisse ihres Aufnahmelandes sowie der komplexen Asylanerkennungsprozeduren. Dies vor allem, wenn sie ihr Herkunftsgebiet überstürzt und weitgehend ungeplant ohne jegliche Vorbereitungen verlassen hatten. Für viele von ihnen bestand das primäre Ziel darin, nach Europa zu gelangen. Erwägungen pro und kontra bestimmter Destinationen erfolgten vielfach erst nach der Ankunft, viele Entscheidungen basierten auf recht vagen Informationen, die während des Verlaufs der Flucht akkumuliert werden konnten, oder waren überhaupt mehr zufällig und unfreiwillig, resultierten also aus zahlreichen Unwägbarkeiten der Flucht und nicht aus strategisch durchdachter Planung. Noch viel weniger als über die jeweiligen Aufnahmeländer generell war dem Gros der Asylwerbenden über regionale Disparitäten innerhalb der Aufnahmeländer, vor allem auf den Wohnungs- und Arbeitsmärkten, sowie über die daraus resultierenden ungleichen Chancen zur strukturellen Integration bekannt. Medial und politisch wurden und werden Geflüchtete aufgrund der sich quantitativ recht deutlich manifestierenden Tendenz zur Bevorzugung bestimmter Aufnahmestaaten innerhalb der EU (z.B. Deutschland, Schweden, Österreich) oftmals mit dem tendenziösen Etikett des „Asylshoppings“ (European Migration Network 2017; Grimm \& BASTAROLI 21-06-2018) versehen. Ein die Vulnerabilität von Geflüchteten verstärkender Generalverdacht! Andererseits soll nicht ausgeklammert werden, dass u.a. McAuliffe \& JAYASURIYA (2016) für einige asiatische Herkunftsstaaten, z.B. auch für Afghanistan, sehr wohl rationale Entscheidungsprozesse von Asylsuchenden für bestimmte Aufnahmestaaten nachgewiesen haben (vgl. dazu auch RoBINson \& SEGROTT 2002).

Aufgrund des transitorischen Charakters und der langen Dauer der Flucht zahlreicher Menschen ist es besonders schwierig, ein valides Bild der sich permanent wandelnden Räumlichkeit sowie der räumlichen Teilaspekte von umfassenderen Fluchtprozessen und individuellen Fluchtbiographien zu zeichnen und exakt festzustellen, welche ökonomischen, politischen und gesellschaftlichen Push- und Pull-Faktoren hierfür in welchem Ausmaß verantwortlich sind. Während reichlich Analysen zur ungleichen Verteilung von Geflüchteten auf die und in den verschiedenen EU-Mitgliedsstaaten vorhanden sind (vgl. Altemeyer-Bartscher 2016; BreKke \& AARset 2009; Rogelu 2017; Thielemann et al. 2010 etc.), ist erheblich weniger über die Muster der sekundären Migration innerhalb der einzelnen Nationalstaaten bekannt. Dies betrifft die Binnenmigration allgemein, die interregionale Migration sowie die Land-Stadt-Migration. Außer Zweifel steht, dass die größeren Städte überall in Europa die Hauptdestinationen für die Geflüchteten sind und somit auch die Herausforderungen der Integration zu tragen haben. Dies galt für die Zeit der „refugee crisis“ ebenso wie für die aktuelle Situation, 
in der Geflüchtete im Rahmen einer Binnenmigration nach wie vor in die Metropolen streben (EuroCities 2016; KonRad AdENAUER Foundation 2016).

Sind die Geflüchteten (auf welche Weise auch immer) einmal in einem bestimmten Aufnahmestaat ,gelandet“, so erfolgt die Verteilung in einigen europäischen Staaten gemäß bestimmten länderspezifischen sowie regionalen Verteilungsschlüsseln (vgl. unten). Viele Geflüchtete stammen jedoch aus urbanen Kontexten, oftmals aus großstädtischen Agglomerationen ihrer Herkunftsländer. Nicht wenige werden aber in Österreich wie in anderen EU-Staaten in Asylquartieren in Kleingemeinden in besonders peripheren und verkehrsmäßig schlecht erschlossenen Lagen untergebracht. Abgesehen von der sozialen und ökonomischen Vulnerabilität der Asylwerber/innen generell tritt als zusätzliche Aspekt also das organisatorisch und institutionell erzwungene Leben in der Seklusion von Asylquartieren an häufig abgelegenen Standorten hinzu. Dies war in der Vergangenheit aufgrund langer Asylverfahren vielfach über Zeiträume von einigen Monaten oder sogar Jahren der Fall. (Die Dauer der Asylverfahren in Österreich ist allerdings seit einiger Zeit infolge personeller Aufstockung der relevanten Behörden deutlich rückläufig.) Welche Auswirkungen hat die politisch-administrativ verordnete räumliche Verteilung Geflüchteter während des Asylverfahrens aber auf die Vulnerablität derselben im oft ländlichen Aufnahmekontext? Für die meisten Geflüchteten stellt dies eine erhebliche zusätzliche psychosoziale Belastung und einen Stressor dar, was jede/r nachvollziehen kann, der/die als Großstädter/in - unfreiwillig und nicht zum Zweck der Erholung - längere Zeit an Orten verbrachte, wo medizinische Infrastruktur, Zugang zu Bildungsangeboten, Schulbesuch für Kinder sowie Grundversorgung mit Waren des täglichen Bedarfs erhebliche Anforderungen an Mobilität und Erreichbarkeit darstellen. Während Einheimische die weiten Wege in der Regel mit dem PKW zurücklegen, steht diese Alternative Geflüchteten so gut wie gar nicht offen. Sie sind auf das dünne Netz des ÖPNV (Öffentlichen Personennahverkehrs) im ländlichen Raum angewiesen. Hinzu kommt das Fehlen einer ethnischen Community, die in Form von Informationsdiffusion über Arbeits- und Wohnmöglichkeiten auch vielfältige praktische Integrationshilfestellungen bieten könnte. Kontakte zu bereits in Österreich lebenden Angehörigen sind nur eingeschränkt möglich (gesetzlich erlaubte bis zu dreitägige Besuche und vor allem über Skype und Social Media). Finanzielle Ressourcen für gegenseitige Besuche fehlen allerdings oftmals. Dass sich räumliche Peripherität auch nachteilig auf die soziale Integration auswirken kann, aber nicht unbedingt muss, ist naheliegend. Allerdings können diese Defizite in ihrer Summe die Vulnerabilität von geflüchteten Menschen verstärken sowie die Perspektivlosigkeit die Tendenz zu Depressionen und anderen psychischen Erkrankungen steigern, was wiederum die Vulnerabilität erhöht.

Die rechtlichen, institutionellen, politischen und ökonomischen Determinanten sowie Folgewirkungen der räumlichen „Platzierung“von Geflüchteten auf der Bundesländerebene, aber auch im Spannungsfeld zwischen Stadt und Land spielen zwar eine wichtige Rolle, sollen aber nicht primär im Fokus dieses Beitrags stehen. Denn bislang ist nur wenig empirisch Gesichertes über die Perspektiven der Geflüchteten selbst, ihre individuellen Wohnstandortpräferenzen, die Motive für die Land-Stadt-Binnenmigra- 
tion sowie Entscheidungsstrukturen in Richtung geplanter Wohnsitzverlegungen innerhalb Österreichs bekannt. Fragen dazu wurden im Rahmen einer 2017/18 vom Bundesministerium für Europa, Integration und Äußeres (bmeia) finanzierten empirischen Untersuchung gestellt (vgl. Datengrundlagen): Welche Begründungen werden für die Attraktivität der Metropolen ins Treffen geführt? Inwieweit spielen unterschiedliche Determinanten der Vulnerabilität eine Rolle für die Binnenmigrationsentscheidung? In welchem Ausmaß sind Erfahrungen mit Xenophobie relevant, wenn Geflüchtete sich entscheiden in die Stadt zu ziehen? Wie beurteilen die interviewten Geflüchteten selbst die soziale und strukturelle Integration in ihrer Unterbringungsgemeinde bzw. späteren Wohngemeinde und -region? Welche Integrationschancen sehen sie für sich in der Großstadt? Welche Rolle spielen die Bildungschancen der Kinder im Rahmen der Wohnstandortentscheidungen? Wie beeinflusst die eigene Herkunft aus dem urbanen Kontext im Herkunftsland das Binnenmigrationsverhalten?

Es sollen also die persönlichen Erfahrungen und Bewertungen der Geflüchteten und Expert/inn/en, ihre Sicht der Push- und Pull-Faktoren sowie die Beurteilung ihrer Integrationschancen am jeweiligen Wohnstandort im Vergleich zu den erhofften Chancen in urbanen Zentren (v.a. Wien) analysiert werden. Der zeitliche Bezugspunkt ist jedenfalls der Zeitpunkt der Durchführung der Befragung.

\section{Freiwillige versus erzwungene Binnenmobilität: Motive und Auswirkungen von Dispersionspolitiken}

Binnenmigration von Immigrant/inn/en kann auf eigenen Entscheidungen basieren oder durch politisch-rechtliche Rahmenbedingungen erzwungen werden. Einige europäische Staaten wurden infolge ansteigender Zahlen von Asylsuchenden mit dem Phänomen des ausgeprägten regionalen Clusterings konfrontiert. Die nationalen Verteilungsstrukturen der Geflüchteten wurden immer komplexer. Dies führte aber auch zur Verstärkung der ethnischen Wohnsegregation in den Agglomerationen (vgl. MALMBERG 2016) und konfrontierte vor allem die großen Metropolen mit unverhältnismäßig starker Zuwanderung, damit einhergehenden steigenden Sozialausgaben und Verknappungen des Angebots auf den für Asylberechtigte legistisch und finanziell zugänglichen Teilwohnungsmärkten (DANZER \& YAMAN 2013; RoBInson et al. 2003).

Zwingende Verteilungsstrategien für neu ankommende Asylwerber/innen, die Konzentrationen in den dicht besiedelten Metropolitan Areas zu verhindern beabsichtigen, sind in Deutschland in Kraft. Als eine Konsequenz der Integrationsherausforderungen entschieden sich weitere europäische Staaten (Schweden, Dänemark, Norwegen, UK und Irland) dazu, politisch motivierte Strategien der Flüchtlingsverteilung zu konzipieren. Ein frühes Modell des geographischen Clusterings von Geflüchteten aus den östlichen Landesteilen Deutschlands wurde bereits 1949 eingeführt, um eine relativ 
gleichmäßige Verteilung von Vertriebenen auf die unterschiedlichen Regionen/Länder der BRD zu erzielen (Boswell 2003). Dieses System trug die Bezeichnung „Königsteiner Schlüssel“ und basierte auf einem Quotenschlüssel nach der Bevölkerungszahl des jeweiligen Bundeslandes sowie dem Steueraufkommen. Es ist immer noch in Geltung und soll gewährleisten, dass kein Land über seine Kapazitäten hinaus beansprucht wird. Regionale oder munizipale Instanzen oder Entscheidungsträger spielten keine Rolle in den Entscheidungsprozessen (ThiELEManN et al. 2010). Das heißt, die deutsche Dispersionspolitik wird großteils geleitet von den Mitteln der öffentlichen Hand, welche Asylwerbende in der ihnen zugewiesenen Lokation erhalten. Entscheiden sie sich dafür, ihren Wohnstandort zu verlegen, so verlieren sie den Anspruch auf öffentliche Unterstützungen und müssen ihren Lebensunterhalt aus eigenen Ressourcen bestreiten (DAHLBERG \& VALEYATHEEPILlay 2018).

In den Niederlanden wurde eine Dispersionspolitik von 1950 bis 1992 verfolgt. Diese Politik implementierte in vielen Städten eine sogenannte ,five percent immigrants regulation“ (RoBINSON et al. 2003). Außerhalb Europas waren es Kanada und die USA, aber auch andere, welche innerstaatliche Dispersionsstrategien verfolgten. Die primären Ziele dieser politischen Strategien bestanden darin, die geographische Verteilung Geflüchteter zu lenken (Andersson 2003; PiIl Damm \& Rosholm 2005) und auf diese Weise deren Arbeits- und Wohnungsmarktintegration positiv zu steuern.

1999 wurde im UK eine Dispersionspolitik in Kraft gesetzt. Diese bezieht sich auf Geflüchtete und andere Asylwerbende, die ökonomisch von Leistungen der öffentlichen Hand abhängig sind, und nimmt keine Rücksicht auf persönliche Präferenzen. Geflüchtete werden auf Regionen mit ausreichendem Wohnungsbestand aufgeteilt, wobei auch andere strukturelle Integrationsrahmenbedingungen vorhanden sein müssen (PHILLIPS 2006; RoBInson et al. 2003). Die Rechtsgrundlage bietet der Asylum and Immigration Act (1999), wobei die Binnendispersionsstrategien im UK im Rahmen einer generellen Privatisierungsstrategie organisiert wurden (DARLING 2016; Hynes 2011). In Irland ist eine Verteilungsstrategie Geflüchtete betreffend im Jahr 2000 in Kraft gesetzt worden (DAHLBERG \& VALEYATHEEPILlay 2018). In Frankreich involviert die räumliche Binnenallokation von Geflüchteten sowohl die nationale als auch die diversen lokalen administrativen Ebenen.

In Schweden wurde die sogenannte „All-of-Sweden“-Strategie 1985 implementiert und bis 1994 beibehalten (RoBInson et al. 2003). Diese Politik platzierte Asylwerbende ohne Rücksicht auf persönliche Präferenzen in Flüchtlingsunterkünften, welche über das ganze Land verteilt waren (ÅsLUND et al. 2009) und in den jeweiligen Kommunen nach Herkunftssprachen diversifiziert wurden (EDIN et al. 2003). Das Hauptziel dieser Strategie manifestierte sich darin, den Flüchtlingsstrom von den metropolitanen Räumen abzuleiten und eine gewisse Balance zwischen urbanen und ruralen Raumeinheiten zu erzielen. Die Bedürfnisse der Geflüchteten wurden zwar berücksichtigt und ihnen sollte es auch nach dem Asylentscheid möglich sein, am betreffenden Wohnort zu verbleiben und sich dort in das Bildungssystem und/oder den lokalen Arbeitsmarkt zu integrieren (ANDERSSON \& MEKKONEN 1996). Recht bald manifestierten sich aber 
in zahlreichen Kommunen erhebliche Verknappungen auf den lokalen Wohnungsmärkten, sodass die verfügbaren Ressourcen auf dem Wohnungsmarkt zu der wesentlichsten Determinante für die Verteilung der Geflüchteten wurden. Ein grundsätzlicher kontradiktorischer Gegensatz zwischen den Wohnpräferenzen der meisten Geflüchteten und den verfügbaren Ressourcen war nicht aufzulösen: Während das Gros der Geflüchteten eine Präferenz für die Metropolen artikulierte, waren gerade dort die verfügbaren Ressourcen an leistbarem Wohnraum durchwegs am knappsten (ÅsLund et al. 2009).

Im derzeitigen schwedischen Migrationssystem spielt die lokale Ebene eine Schlüsselrolle, denn die Kommunen verfügen über weitgehende autonome Entscheidungskompetenzen darüber, bestimmte Geflüchtete aufzunehmen oder auch abzulehnen. Dies hat inzwischen zu beträchtlichen räumlichen Disparitäten hinsichtlich der lokalen Aufnahmebereitschaft gegenüber Geflüchteten und in der Folge bezüglich der Zahlen an faktisch aufgenommenen Asylsuchenden geführt (LidÉN \& NYHLÉN 2013; LUNDGREN 2018). Die räumliche Umverteilungspolitik soll primär - wenn nicht sogar ausschließlich - der Arbeitsmarktintegration dienlich sein (AldÉN \& HAMMARSTEDT 2014; DAmm \& Rosholm 2005). In der Realität zeigt sich allerdings, dass die räumliche Dispersion der Geflüchteten eher das Resultat spezifischer lokaler Faktorenkonstellationen und nicht ausschließlich der Möglichkeiten, die sich in den beiden wichtigsten strukturellen Integrationsbereichen des Arbeitens und Wohnens bieten, ist. Zielorientierte Strategien und geplante Maßnahmen erreichen hierbei nicht immer den intendierten Zweck (AldÉN \& HammarstedT 2014).

Dänemark verfolgte von 1986 bis 1998 eine Politik der innernationalen Verteilung von Asylberechtigten, die unter Leitung und Supervision des Danish Refugee Council stand (DAmm \& Vasiljeva 2016). Das wesentliche Ziel manifestierte sich darin, die Geflüchteten je nach der Bevölkerungszahl von politischen Bezirken und (Stadt-) Gemeinden im Land gleichmäßiger zu verteilen. Die dänische Vorgangsweise war der schwedischen sehr ähnlich: Sie bezog sich auf das gesamte Staatsterritorium. (Stadt-) Gemeinden, in denen Geflüchtete untergebracht wurden, mussten über die dazu nötige Infrastruktur verfügen. Es war wie in Schweden Geflüchteten grundsätzlich gestattet, in andere Gemeinden zu übersiedeln. Unabdingbare Voraussetzung hierfür war aber, dass die Betreffenden eigeninitiativ eine Unterkunft (z.B. bei Angehörigen) gefunden hatten. Individuelle Lokalisationspräferenzen fanden keine Berücksichtigung und ebenso konnten sich die Gebietskörperschaften nicht „bestimmte“ Geflüchtete (z.B. Höherqualifizierte, Personen mit Ausbildungen in Mangelberufen) aussuchen (DAHLBERG \& VALEYATHEEPILLAY 2018).

Resümierend ist festzustellen: Dispersionspolitische Strategien sind durchaus imstande, positive Effekte im Hinblick auf die Kostenverteilung der Integration von Geflüchteten zu erzielen, ebenso eröffnen sie Möglichkeiten der mittel- und langfristigen Integration auch in für Immigrant/inn/en auf den ersten Blick weniger attraktiven ländlichen Regionen, sofern diese als Wirtschaftsstandorte über Arbeitskräftenachfrage verfügen. Zugleich vermindern sie den Druck auf lokale Wohnungs- und Arbeitsmärkte. Indem sie darüber hinaus ethnische Segregationstendenzen hintanhalten, können sie 
auch dem Erlernen der jeweiligen Landessprache förderlich sein und auch auf der submunizipalen Ebene (z.B. im räumlichen Kontext der lokalen Neighborhoods) wirken (DAhlberg \& Valeyatheepillay 2018, 6).

Negativ zu bewerten ist, dass diese Politiken ausschließlich obrigkeitsstaatlichen Kriterien entsprechen und mit Zwang durchgesetzt wurden/werden. Somit wird keine Rücksicht auf für die soziale Integration so wichtige bereits existente soziale Netzwerke der Immigrant/inn/en genommen. Faktoren wie verkehrsmäßige Erreichbarkeit sowie die regelmäßige Verfügbarkeit von Orten für gemeinsames Gebet, aber auch kulturelle und soziale Zusammenkünfte werden ebenfalls nicht in Erwägung gezogen. Die Wohnstandortentscheidung soll ausschließlich rationalen, sachlogischen Überlegungen hinsichtlich rascher Arbeitsplatzfindung und der Verfügbarkeit und „Auffüllung“ vakanten Wohnraums erfolgen. Dass die ethnische Diaspora wichtige Funktionen für alle Bereiche der Integration erfüllt, aber in ländlichen Regionen weitgehend fehlt, findet keine Berücksichtigung.

\section{Wissenschaftlicher State of the Art}

Bislang wurde den mittel- und längerfristigen Konsequenzen der dispersen regionalen Verteilung von Geflüchteten innerhalb nationalstaatlicher Grenzen nur beschränkte wissenschaftliche Aufmerksamkeit zuteil (FrATESI 2016). Dies gilt auch für die Entscheidungsprozesse und individuellen Mobilitätspräferenzen, die für die Sekundärmigration in den europäischen Aufnahmestaaten verantwortlich zeichnen (u.a. Åslund 2005; Birgier et al. 2016; Lindley \& VAN HeAR 2007; Robinson \& SEGRotT 2002). Größeres Forschungsinteresse wurde diesen Fragen in den USA (z.B. BLoEM \& LOVERIDGE 2017, 2018), aber auch in anderen klassischen Einwanderungsländern wie Kanada und Australien gewidmet.

Das beschränkte wissenschaftliche Interesse ist vor allem darauf zurückzuführen, dass nur wenig tragfähiges empirisches Material vorliegt, welches als Evidenz für individuelle Mobilitätsentscheidungsprozesse und Wohnstandortpräferenzen sowie deren Determinanten herangezogen werden könnte. Die vorhandenen wissenschaftlichen Analysen fokussieren in erster Linie auf die Arbeitsmarktintegration und deren Herausforderungen (AldÉn \& HammarstedT 2014). Den lokalen Wohnungsmärkten wurde im Zusammenhang mit Binnenmigration von Geflüchteten bislang viel weniger Aufmerksamkeit geschenkt (ABRAHAMsSON 2002), obwohl zahlreiche Kommunen vor nicht minder schwer zu lösende Probleme gestellt wurden, wenn es galt quasi simultan temporär nutzbare Quartiere für den Zustrom von Asylwerber/inne/n sowie längerfristige Wohnmöglichkeiten für Asylberechtigte bereitzustellen. Dazu kommt, dass die Produktionszyklen auf dem Wohnungsmarkt ungleich langfristiger sind als jene oft kurzfristigen Jobkonjunkturen in bestimmten Branchen (z.B. Bauwesen, Tourismus) und Subsegmenten auf den regionalen Arbeitsmärkten. Die Determinanten der Wohnsitz- 
und somit Binnenmigrationsentscheidungen sowie die Rolle regionaler Disparitäten hat DAMm (2009b) untersucht. Wie ZaVodny (1999) sowie Zorlu \& Mulder (2008) nachwiesen, handelt es sich um komplexe individuelle Entscheidungsprozesse, was KeLLY \& Hedman (2016) im Zusammenhang mit den Motiven für Sekundärmigration bei hochqualifizierten Immigrant/inn/en aus dem Iran bestätigten.

Ohne an dieser Stelle auf die zahlreichen theoretischen Analysen zu Aspekten der „vulnerability“ eingehen zu können, sei nachstehend aus Veranschaulichungsgründen eine Klassifikation der wesentlichen Determinanten sowie der unterschiedlichen Manifestationen von Vulnerabilität bei geflüchteten Personen (aus SABATES-WHEeler \& WaITE 2003, p. 13) angeführt (vgl. Abb. 1), auf welche im empirischen Teil Bezug genommen wird:

Abb. 1: Determinanten und Manifestationen von Vulnerabilität bei Geflüchteten

\begin{tabular}{|c|c|c|c|c|}
\hline \multirow{2}{*}{$\begin{array}{l}\text { Determinant } \\
\text { of } \\
\text { vulnerability }\end{array}$} & \multicolumn{4}{|c|}{ Examples of manifestations } \\
\hline & $\begin{array}{l}\text { Displacement- } \\
\text { specific }\end{array}$ & Intensified & $\begin{array}{c}\text { Bureaucratically } \\
\text { imposed }\end{array}$ & $\begin{array}{l}\text { Over- } \\
\text { representation }\end{array}$ \\
\hline $\begin{array}{l}\text { Spatial / envi- } \\
\text { ronmental }\end{array}$ & $\begin{array}{l}\text { Restrictions } \\
\text { on movement } \\
\text { (e.g. encamp- } \\
\text { ment). } \\
\text { Unfamiliarity } \\
\text { with surroun- } \\
\text { dings (unsure } \\
\text { about rights } \\
\text { and access). } \\
\text { Increase in } \\
\text { gender-based } \\
\text { violence } \\
\text { Family Split }\end{array}$ & $\begin{array}{l}\text { Lack of know- } \\
\text { ledge (of e.g. } \\
\text { rights, opportuni- } \\
\text { ties for transport) } \\
\text { Double intensity } \\
\text { for women and } \\
\text { dependents }\end{array}$ & $\begin{array}{l}\text { Use of local } \\
\text { languages (can't } \\
\text { understand access } \\
\text { rules) }\end{array}$ & $\begin{array}{l}\text { Health risks asso- } \\
\text { ciated with infor- } \\
\text { mal settlements } \\
\text { (difficult to spend } \\
\text { time accessing). }\end{array}$ \\
\hline $\begin{array}{l}\text { Socio- } \\
\text { political }\end{array}$ & $\begin{array}{l}\text { Lack of } \\
\text { representation } \\
\text { (ineligible to } \\
\text { access, camps } \\
\text { run without } \\
\text { representation) }\end{array}$ & $\begin{array}{l}\text { Uncertainty } \\
\text { interacting with } \\
\text { govt/agencies } \\
\text { (opaque instituti- } \\
\text { onal complaints } \\
\text { mechanisms) }\end{array}$ & $\begin{array}{l}\text { Discrimination in } \\
\text { access to servi- } \\
\text { ces; (ineligible to } \\
\text { access). } \\
\text { Gender-bias in } \\
\text { legislation }\end{array}$ & $\begin{array}{l}\text { Restrictions on } \\
\text { political activi- } \\
\text { ties in camp esp. } \\
\text { for women and } \\
\text { youth (inability to } \\
\text { express voice). }\end{array}$ \\
\hline Socio-cultural & $\begin{array}{l}\text { Xenophobia } \\
\text { (discrimination } \\
\text { in access) }\end{array}$ & $\begin{array}{l}\text { Social discrimi- } \\
\text { nation based on } \\
\text { ethnicity, langua- } \\
\text { ge, illegal status, } \\
\text { gender and age } \\
\text { (discrimination in } \\
\text { provision). }\end{array}$ & $\begin{array}{l}\text { Additional stig- } \\
\text { matising require- } \\
\text { ments to access } \\
\text { services (required } \\
\text { to show additional } \\
\text { eligibility docu- } \\
\text { ments) }\end{array}$ & $\begin{array}{l}\text { Social percep- } \\
\text { tions of 'criminal } \\
\text { poor' (additional } \\
\text { scrutiny of access } \\
\text { documents) }\end{array}$ \\
\hline
\end{tabular}

Quelle: Sabates-WheELER and Waite (2003, p. 13). 
In Österreich haben sich die Divergenzen zwischen Wohnungsangebot und -nachfrage in den Landeshauptstädten, vor allem aber in Wien, in der letzten Dekade sukzessive verschärft. Zugleich sind alle österreichischen Städte bestrebt, ethnische Segregationstendenzen zu verhindern, denn über deren negative Auswirkungen für die Integration von Immigrant/inn/en sind sich internationale Expert/inn/en weitgehend einig (ANDERSSON et al. 2010; MARCiŃCZAK et al. 2015; Musterd 2020; MusTERD \& VANKEMPEN 2006). HAJEK (2017) legte eine erste größere Studie unter Geflüchteten zu deren Hauptmotiven für die ausgeprägte Binnenmigration nach Wien vor. KoRDEL et al. (2018) erforschten die Lebenswelten geflüchteter Menschen in ländlichen Regionen qualitativ, wobei auch die vielfältigen Motive für deren Verbleib in Kleingemeinden untersucht wurden.

Die Erfahrungen vor sowie unmittelbar nach dem Asylbescheid bzw. nach der Zuerkennung eines subsidiären Schutzstatus, wie ihn vor allem afghanische Geflüchtete häufig erhalten, sind diesbezüglich oftmals entscheidend, aber auch zu einem späteren Zeitpunkt werden in zahlreichen Fällen noch Wohnstandortwechsel vollzogen, wie die in den nachfolgenden Abschnitten analysierten empirischen Befunde dokumentieren. Die kontroversielle und oftmals mehr von ideologischen als von integrationschancenorientierten Argumenten getragene Diskussion um die Dispersion von Geflüchteten hat in den letzten beiden Jahren infolge der starken Rückgänge bei den Asylwerber/innenzahlen $^{1}$ erheblich an Brisanz verloren. Zahlreiche Asylunterkünfte sowohl in urbanen als auch in ländlichen Kontexten in allen Bundesländern wurden infolge Unternutzung geschlossen. $^{2}$

\section{Zur räumlichen Dispersion und Binnenmigration von Geflüchteten in Österreich}

Für Österreich ist prinzipiell zwischen zwei Phasen zu unterscheiden, in welchen hinsichtlich der individuellen Möglichkeiten Geflüchteter zu räumlicher Mobilität völlig unterschiedliche legistische Normen gelten: 1) Asylwerber/innen/n während der Phase des Asylverfahrens und 2) Asylberechtigte (bzw. subsidiär Schutzberechtigte)

1 Im Spitzenjahr 2015 suchten 88.340 Personen (25.563 Afghan/inn/en) um Asyl an (BM.I, 2018, 4). Danach gingen die Zahlen stark zurück, nämlich auf 42.285 (11.794 Afghan/inn/en) Anträge 2016, 24.735 im Jahr 2017 (3.781 von Bürger/inne/n Afghanistans) und 13.746 (2.120 Afghan/inn/en) im Jahr 2018 (BM.I, 2015, 2016, 2017, 2018, 2019a). Anmerkung: Die Jahresstatistik 2019 stand zum Zeitpunkt der Erstellung dieses Beitrags noch nicht zur Verfügung.

2 Seit dem Höchststand 2015 mit 530 Unterkünften sind bis Mitte 2019 beispielsweise in Oberösterreich fast 300 Flüchtlingsheime geschlossen worden. Die Zahl der neuen Asylanträge ging 2019 kontinuierlich zurück, weshalb etwa in den Monaten Mai und Juni 201915 weitere Asylunterkünfte aufgelassen wurden (orf, 04-05-2019). Analoge Entwicklungen vollzogen sich in allen Bundesländern. 
nach Erhalt des Asylbescheids. Der unterschiedliche Rechtsstatus determiniert auch divergierende Verpflichtungen bzw. Rechte in Bezug auf Binnenmigration, also dauerhaften Wohnsitzwechsel innerhalb des Bundesgebiets.

1) Asylwerber/innen werden grundsätzlich in Asylquartieren untergebracht und auf alle Bundesländer verteilt, wo sie auch Grundversorgung erhalten. Demnach leistet der Bund im Wesentlichen die Grundversorgung für Asylwerber/innen in der ersten Phase des Asylverfahrens, dem sogenannten Zulassungsverfahren. In dieser Zeit werden die Asylwerber/innen grundsätzlich in Bundesbetreuungsstellen untergebracht und versorgt. Es gibt Bundesbetreuungsstellen bei den Erstaufnahmestellen, bei Verteilerquartieren, Bundesbetreuungsstellen für Menschen mit besonderem Betreuungsbedarf sowie bedarfsweise Notquartiere. Grundsätzlich werden in die Erstaufnahmestellen nur mehr jene Asylwerber/innen zugewiesen, für deren Asylantrag voraussichtlich ein anderer Mitgliedsstaat zuständig ist. Jene, für deren Asylantragsprüfung voraussichtlich Österreich zuständig ist, werden nicht mehr - wie in der Vergangenheit - in die Erstaufnahmestellen zugewiesen, sondern es erfolgt eine Aufteilung auf die Verteilungsquartiere des Bundes in den Bundesländern entsprechend der Quote nach der Grundversorgungsvereinbarung - Art. 15a B-VG (vgl. BMI/Bundesministerium für Inneres 2019b). Da vor allem in Zeiten hoher Fluchtimmigration einige Bundesländer die Quoten nur unzureichend erfüllten, war die Verteilung immer stark wienlastig (vgl. FABRY 19-07-2018). Verteilerquartiere befinden sich in Kreuzen (Oberösterreich), Wien, Nussdorfer Straße (zuständig für Wien und das Burgenland), Traiskirchen (Niederösterreich), Bergheim (Salzburg), Innsbruck (zuständig für Tirol und Vorarlberg), Graz-Puntigam (Steiermark) und Ossiach (Kärnten). ${ }^{3}$

Ab dem Zeitpunkt der Zulassung, bzw. Entscheidung, den Asylantrag inhaltlich hinsichtlich des Fluchtvorbringens zu prüfen, geht die Zuständigkeit der Unterbringung und Versorgung der Asylwerber/innen auf die Länder über. Bei einer solchen Zulassung zum Asylverfahren sollten Asylwerber/innen von den Bundesländern aus den Verteilerquartieren des Bundes so rasch wie möglich in ein Landesquartier übernommen werden. Bei Landesquartieren handelt es sich grundsätzlich um kleinere Quartiereinheiten (beispielsweise ehemalige Pensionen, Heime etc.) im Zuständigkeitsbereich der Bundesländer. Der Abschluss der entsprechenden Verträge mit den Quartiergebern fällt in den Zuständigkeitsbereich des jeweiligen Bundeslandes. ${ }^{4}$ Die Geflüchteten haben sich an diesen Örtlichkeiten aufzuhalten und den am jeweiligen Ort verantwortlich zeichnenden Organisationen zur Verfügung zu stehen. Mit 1. November 2017 ist eine Gesetzesänderung in Kraft getreten, die Asylwerber/inne/n einen Wohnsitzwechsel in ein anderes Bundesland nicht mehr erlaubt. Im Falle ungemeldeten und somit unautorisierten Ortswech-

$3 \quad$ Vgl. $<$ https://www.bmi.gv.at/303/start.aspx > [Zugriff 22-01-2020].

4 Vgl. ebenda. 
sels kann eine Geldstrafe verhängt werden. ${ }^{5}$ Während des Asylverfahrens kommt es also in jedem Fall zu einer räumlichen Dispersion der betreffenden Geflüchteten. Denn die Entscheidung, in welchem Bundesland eine geflüchtete Person sich während des Asylverfahrens aufzuhalten hat, wird ohne Rücksicht auf die Vulnerabilität der Person vom Innenministerium getroffen (vgl. den Beitrag ArSLAN, Balbous \& MACH in diesem Band).

Im 50-Punkte-Plan zur Integration von Asylberechtigten und subsidiär Schutzberechtigten in Österreich des EXPERTENRATES FüR INTEGRATION (2015, p. 23) heißt es bezüglich der Verteilung im Bundesgebiet unter Punkt 44: „Gleichmäßige regionale Verteilung von Flüchtlingen: Um die Integration von Asylberechtigten bzw. subsidiär Schutzberechtigten möglichst rasch zu ermöglichen, muss eine verstärkte Ansiedlung der Zielgruppe in ländlichen Regionen sowie eine ausgeglichene Verteilung innerhalb Österreichs angestrebt werden. Dies hat mehrere Gründe, u.a. die Tatsache, dass die Wohnraumpreise in Ballungsräumen stark angestiegen sind und weiter ansteigen werden, was der Zielgruppe nicht zuträglich ist. Weiters ist überdies bekannt, dass Integration in der unmittelbaren Nachbarschaft rascher funktioniert als in größeren städtischen Gebieten, da leichter soziale Kontakte geknüpft werden.“ Dass dieses Ziel mit den aktuell zur Verfügung stehenden legistischen Möglichkeiten nicht erreicht werden kann, steht außer Frage. Dies wird umso klarer, wenn man die in den narrativen Interviews der vorliegenden Befragung getätigten Aussagen zu den Wohnsitzpräferenzen im urbanen Raum einbezieht.

2) Anerkannte Asylberechtigte und subsidiär schutzberechtigte Personen können ihren Aufenthaltsort eigenbestimmt wählen, obwohl 2016/17 mediale und politische Diskussionen über die etwaige Installierung räumlicher Dispersionsstrategien auch in Österreich geführt wurden. Dies war auf die damals hohen Zahlen an Geflüchteten zurückzuführen, welche nach nachhaltigen Lösungen einer gleichmäßigeren Verteilung im Bundesgebiet suchen ließ. Letztlich wurden aber formal keine politischen Maßnahmen in diese Richtung beschlossen. Anstatt dessen wurde und wird nach wie vor versucht, auf einer freiwilligen Basis über die Informationen zu Arbeitsmarktchancen und intensive Beratung eine Dispersion zu erzielen. Die Wirtschaftskammer Österreich sowie das Arbeitsmarktservice spielen hier eine entscheidende Rolle. So stimulierte die WKO arbeitsangebotsbasierte Strategien über die systematische Informationsbereitstellung zu nachhaltigen Beschäftigungschancen auf regionalen Teilarbeitsmärkten und in bestimmten Branchen, vor allem im Tourismus und in anderen „Mangelberufen“, die explizit definiert und aufgelistet wurden. ${ }^{6}$ Seit 2019 existieren zwei Listen für Mangelberufe, eine

Vgl. Diakonie FlüChtLingSDIENST, Informationsblatt für Klient*innen zur Wohnsitzbeschränkung <https://www.asyl.at/aduploads/141.04.ma,wohnsitzbeschrnkungdeutsch.pdf $>$ [Zugriff 26-01-2020]. 2019].

Vgl. <https://www.wko.at/site/Migration/fachkraefte_mangelberufe.html> [Zugriff 03-04- 
nationale sowie eine bundesländerspezifische. ${ }^{7}$ Diese Listen werden jährlich revidiert und aktualisiert. Zusätzlich hierzu wurde der Zugang von potentiellen Lehrlingen mit Fluchthintergrund zu spezifischen Lehrberufen und Branchen durch die Wirtschaftskammern gefördert und erleichtert, um dem akuten bzw. chronischen Mangel an Lehrlingen in bestimmten Lehrberufen gezielt entgegenzuwirken. Im Rahmen der neuen Regierungskoalition 2020 hat die zuständige Bundesministerin für Digitalisierung und Wirtschaftsstandort explizit Verschärfungen der Zumutbarkeitsbestimmungen hinsichtlich der regionalen Vermittlung von (jungen) Asylberechtigten angekündigt. Diese sollen beschäftigungslose, beim AMS vorgemerkte und „lokal nicht verwurzelte ...“ (Szigetvari 22-01-2020) Asylberechtigte „... ohne Betreuungspflichten“ betreffen, die von Wien in die westlichen Bundesländer mit größerem Arbeitsplatzangebot vermittelt werden sollen.

Statistisches Faktum ist, dass sich im langjährigen Vergleich mehr als 40\% des „migration inflows“ nach Österreich in der Bundeshauptstadt Wien konzentriert und selbige zur wichtigsten ,arrival city“ für sogenannte Drittstaatsangehörige machen, eine Kategorie, die v.a. Geflüchtete und andere Asylberechtigte inkludiert (vgl. KoHLBACHER \& REEGER 2020). Obwohl tragfähige offizielle Zahlen zur Binnenmigration von Asylberechtigten nicht verfügbar sind, kann angenommen werden, dass die überwiegende Mehrheit der Afghan/inn/en nach dem positiven Asylbescheid aus den Bundesländern nach Wien übersiedelt. Diese These kann durch Befragungsresultate aus Erhebungen des Instituts für Stadt- und Regionalforschung (ISR) untermauert werden, ebenso aber mithilfe von Schätzungen, die sich auf Geflüchtete unterschiedlicher Herkunftsnationen beziehen, und von denen zwischen 60 und 80\% angeben, eine Binnenmigration nach Wien zu planen (ADDENDUm 28-04-2018, p. 4). Bislang spielten hierfür auch die divergierenden Levels der Bedarfsorientierten Mindestsicherung in den einzelnen Bundesländern eine Rolle (MüLlER 2016). Das Ungleichgewicht zwischen dem Ausmaß der Zuwanderung nach Wien und der Neubauleistung auf dem Wohnungssektor kann durch folgende Zahl illustriert werden: Im Jahr 2016 entfielen in Wien auf jede fertiggestellte Neubauwohnung 4,8 Neuzuwanderer/innen, die sich zumeist auf der Suche nach leistbarem Wohnraum befanden (ADDENDum 28-04-2018, p. 5).

Aufgrund einer spezifischen politischen Faktorenkonstellation (Wien als die traditionelle Hochburg der Sozialdemokratie und einer diversitätsorientierten Integrationspolitik; Hoekstra et al. 2018) war die Bundeshauptstadt innerhalb Österreichs auch stets ein spezifisches „Biotop“ für die Integration Geflüchteter (STADT WiEN, MagisTRATSABTEILUNG 17, o.J.). Während in anderen Bundesländern die Asylwerber/innen vielfach in überfüllten Massenquartieren untergebracht wurden, viele davon an äußerst peripheren Standorten, was der Stigmatisierung der Menschen bei der lokalen Bevölkerung oftmals sehr förderlich war (AUMüLLER 2018), verfolgte Wien eine völlig konträre Strategie. Die Verteilung der neuen Asylwerber/innen erfolgte vor allem in kleineren

7 Vgl. AsYlKoORDinATION (2020): <https://www.migration.gv.at/de/formen-der-zuwanderung/dauerhafte-zuwanderung/bundesweite-mangelberufe/ $>$ sowie $<$ https://www.migration. gv.at/de/formen-der-zuwanderung/dauerhafte-zuwanderung/regionale-mangelberufe/>. 
Asylquartieren und privaten Unterkünften, die zudem möglichst über den gesamten Stadtraum verstreut waren, um Konzentrationen zu vermeiden. So lebten etwa im September 2019 in Wien nur 29\% der Geflüchteten in staatlicher Grundversorgung in 57 größeren Asylunterkünften. Hingegen waren $71 \%$ in kleineren privaten Quartieren in verschiedensten Wiener Bezirken untergebracht (Fonds Soziales WiEn, Sept. 2019, p. 2). Hinzuzufügen ist jedoch, dass auch diese Dispersionsstrategie nicht immer konfliktfrei vonstattenging (WIENER ZeITUNG, 14-03-2016).

\section{Empirische Resultate auf Basis der Interviews}

\subsection{Datengrundlagen}

Die empirische Grundlage bildet eine 2017/18 vom Bundesministerium für Europa, Integration und Äußeres (bmeia) finanzierte Befragung unter Geflüchteten aus Afghanistan. Für die Studie wurden 65 Respondent/inn/en (27 weibliche, 38 männliche) mit positivem Asylbescheid oder subsidiärem Schutz interviewt, die zum Zeitpunkt der Erhebung in Wien, Nieder- bzw. Oberösterreich wohnhaft waren. Die Auswahl erfolgte gemäß den Variablen Ankunftsjahr in Österreich (nach den Kategorien 1970er-, 1980er-, 00er-/10er- Jahre, 2013/2014 und ab 2015), Alter, ethnische Zugehörigkeit, Bildungsstatus und Beruf zum Erhebungszeitpunkt.

13 Expert/inn/en aus relevanten Organisationen wurden ebenfalls befragt. Darunter befanden sich Mitarbeiter/innen von beratenden Organisationen (z.B. Beratungszentrum für Migranten und Migrantinnen und der ÖIF/Österreichische Integrationsfonds), aus Behörden wie der Magistratsabteilung 17 - „Integration und Diversität“, teils Expert/inn/en, die selbst ehemalige Asylwerber/innen waren, sowie Repräsentant/inn/en afghanischer Kulturvereine. Die politische Perspektive wurde anhand eines Interviews mit einem zuständigen Landesrat erhoben.

Das Interviewer/innenteam umfasste vier Projektmitarbeiter/innen sowie sechs eigens angeworbene Personen (zwei weiblich, vier männlich) mit muttersprachlichen Dari/Farsi-Kenntnissen und mehrere ethnische Minoritäten abdeckend (Hazara, Pashto, Tadjik, Uzbek). Es wurden in erster Linie muttersprachliche Interviews durchgeführt und auf eine kultursensible Interviewführung Wert gelegt. Bei Gesprächen auf Dari/ Farsi gehörten Interviewer/innen und Respondent/inn/en demselben Geschlecht an. Im Vorfeld der Erhebungsphase erfolgten drei Pretestinterviews. Etliche der bereits lange in Österreich aufhältigen Afghan/inn/en wünschten explizit, in deutscher Sprache befragt zu werden.

Die Schaffung des Kontaktpools erfolgte über Netzwerke der afghanischen Interviewer/innen und Gatekeeper/innen der Community. Sodann wurden mittels der Methode des Schneeballsystems unter Quotakontrolle (Verteilung nach ethnischen 
Gruppen, soziodemographischen sowie bildungsbezogenen Variablen) Respondent/inn/ en akquiriert.

Das Erhebungsinstrument bestand in leitfadenbasierten Interviews. Das Fragenspektrum erstreckte sich von Wohnpräferenzen, Spracherwerb, Arbeits- und Wohnungsmarktintegration, Wertewandel, Religion, über Gender Role Models, Demokratie und Rechtsstaat bis zu persönlichen Erfahrungen mit Institutionen und dem Asylverfahren. Der in deutscher Sprache erstellte Leitfaden wurde sodann übersetzt und einer nochmaligen Sprachüberprüfung unterworfen. Die transkribierten Interviews wurden mittels des computergestützten Analyseprogramms ATLAS.ti anhand einer vorher im Projektteam erstellten Liste codiert und sodann ausgewertet.

Im Rahmen einer Fokusgruppendiskussion am Institut für Kultur- und Sozialanthropologie der Universität Wien konnten vorläufige Ergebnisse kritisch reflektiert und zusätzliche Informationen eingeholt werden.

\subsection{Räumlich-regionale Wohnpräferenzen und Binnenmigration}

In der politischen und medialen Diskussion um die räumlichen Aspekte der Flüchtlingsintegration wurden Modelle durchwegs erfolgreicher Integration der Geflüchteten in durch Abwanderung geprägten Räumen und unterstützt durch lokale Ehrenamtliche sowie in kleineren Gemeinden generell den undifferenziert gezeichneten negativen Auswirkungen der Konzentration ethnischer Communities in urbanen Ballungszentren antithetisch gegenübergestellt. Aus letzterer werden die Gefahr einer ausgeprägten Communitybildung sowie Tendenzen möglicherweise sogar bis zur Etablierung von Parallelgesellschaften in bestimmten Stadtvierteln abgeleitet (Augustin 2012; MANEA et al. 2017). Beispiele für Segregation und deren Auswirkungen finden sich in zahlreichen europäischen Metropolen (MARCiŃCZAK et al. 2015; Musterd 2020).

Diese simplifizierende Sicht bildet allerdings nicht die Realität ab, die de facto vielschichtig ist. Da in Österreich keine dispersionspolitischen Maßnahmen zur räumlichen Verteilung installiert wurden, obliegt es den Geflüchteten selbst die Entscheidung zu fällen, wo sie ihren Wohnsitz wählen wollen. Ohne den politischen Diskurs völlig außer Acht zu lassen, gilt das Interesse dieses Beitrags den Perspektiven der Geflüchteten selbst und diese sollen im Folgenden primär auch zu Wort kommen. Die Entscheidungen sind selbstverständlich von zahlreichen Faktoren auch außerhalb der Sphäre persönlicher Entscheidungsfreiheit abhängig. In Familien müssen die Interessen der Kinder und deren Bildungslaufbahnen berücksichtigt werden. Nicht selten fühlen sich die nichtberufstätigen Frauen in Kleingemeinden ohne ethnische Diaspora isolierter als die arbeitenden Männer. Ganz klar tritt die Präferenz für die Bundeshauptstadt Wien zutage, die auch von den befragten Expert/inn/en bestätigt wird. Aufgrund der großen Fülle an Material konnte nur eine Selektion von weiteren Aspekten der Wohnstandortwahl einer eingehenderen Analyse unterzogen werden: die Rolle von Vulnerabilität, die 
Bedeutung der ethnischen Diaspora sowie der Faktor der Bedarfsorientierten Mindestsicherung.

Für eine nachhaltige Analyse der faktischen Verläufe räumlicher Integrationspfade ist eine einmalige Befragung, wie die in der Erhebung durchgeführte, nicht ausreichend. Zu diesem Zwecke hätte zumindest eine nochmalige Befragung desselben Samples in einem gewissen Zeitabstand erfolgen müssen. Panelbefragungen sind jedoch teuer und aufwendig und in der Forschungsrealität nur in seltensten Fällen umsetzbar. Das Datenmaterial spiegelt aber zumindest eine Momentaufnahme der konkreten Wohnund Aufenthaltspräferenzen in unterschiedlichen Raumtypen wider und lässt zumindest bedingte Rückschlüsse auf das Ausmaß geplanter Wegzüge in die urbanen Agglomerationen (da die Interviews ausschließlich im Osten Österreichs durchgeführt wurden, also vor allem nach Wien) zu.

\subsection{Die Attraktivität des Urbanen: Wien als Zuwanderungsmagnet in Österreich}

Die von Expert/inn/en wie Geflüchteten gleichermaßen betonte Attraktivität der Binnenmigration nach Wien resultiert aus dem Zusammenspiel und Wechselwirkungen zwischen zahlreichen ökonomischen, bildungssystem-, arbeits- und wohnungsmarktbezogenen, aber auch sozialen Faktoren, wie die folgenden Zitate aus Expert/inn/en/ interviews belegen:

„Also viele wollen direkt nach Wien kommen, teilweise weil viele Bundesländer wahrscheinlich denen das Gefühl geben ... und andererseits, weil sich in Wien sehr viel tut. Politisch auch sehr viel tut. Auch gibt es viele Projekte, auch vom Arbeitsmarktbereich. Diese vielen Deutschkurse, alles. Und das ist so zentral und in Wien können sie sich sehr gut bewegen. Also in den Bundesländern bei vielen, die Analphabeten sind und am Land sind, die Herausforderungen ist mal, mit den öffentlichen Verkehrsmitteln irgendwo anzukommen, mit dem Zug oder Bus. Aber in Wien ist das dann so gut mit den Verbindungen, dass dann jeder sich da in Wien gut fühlt. Und zweitens, die Stadt Wien macht ja so viel. Sie arbeiten auf der Ebene von Communities zusammen. Es wird auf den Ebenen, ... also genauso NGO-Bereiche und so, so vieles angeboten, dass die Menschen das Gefühl haben, die haben so viele Informationen und dass sie da weiterkommen. ... Und diese ganzen Projekte, die entstehen, wo der Austausch auch sehr gro $\beta$ ist unter den Menschen ... und natürlich kommen die dann nach Wien. " (Expertin 8)

„Die meisten Jugendlichen, die unbegleitet nach Wien gekommen sind, bleiben auch in Wien. Sie werden auch in Wien betreut, weil sie da bessere Betreuung haben in dem Sinne, dass dieses Jugendcollege auch in Wien ist, wo alleine in 
einem Jahr 1.500 Jugendliche dort Deutschkurse besuchen können, ihre Matura nachmachen können, halt Abschlüsse. Und dann, wenn sie sagen: ,Ok, ich möchte studieren, Matura nachholen', die dann diese drei Jahre begleitet werden, bis sie dann studieren können. Also das sind halt diese Vorteile. “ (Experte 4)

„Die werden, wenn sie einen positiven Bescheid bekommen ..., einerseits sind sie in einer großen Familie groß geworden. Die sind sozialer und die wollen mit den anderen [Afghan/inn/en] etwas zu tun haben, weil von den anderen Einheimischen sind sie nicht aufgenommen worden, nicht akzeptiert und respektiert. Und sie haben ein paar Monate, ein paar Jahre dort [im Asylheim] ausgehalten, haben dann einen positiven Bescheid oder Subsistenzschutz bekommen und sind sofort nach Wien gewechselt und in andere Städte, nach Linz, in Städte umgezogen. Weil zumindest finden die sich dann mit anderen Afghan/inn/en zurecht. Dort haben sie einen Gebetsraum, einen Verein. Dort können sie sich engagieren, haben sie mit anderen zu tun, können sich einander immer wieder sehen, einladen zu sich nach Hause. Bei uns ist das so. Und die bleiben nicht in anderen Städten oder Orten. Die werden nach Wien kommen, in andere Bundesländer, Städte kommen. Es ist nicht die Schuld von den Afghan/inn/en. Viele (Österreicher/innen) sagen: ,Es ist nur wegen dem Sozialgeld: ' Das stimmt auch nicht meiner Meinung nach. Es ist [dass sie in die Städte gehen]), weil sie nicht von der Ortschaft aufgenommen wurden, nicht akzeptiert wurden, nicht in diese Ortschaft zumindest integriert wurden. Eine alte Dame, ein alter Herr braucht das, mit den Menschen zu reden. Die wollen unbedingt reden, weil die Kinder von ihnen sind in den Städten oder in anderen Ländern zum Arbeiten, die haben keine Zeit. “ (Experte 2)

„Eigentlich hauptsächlich, einen Job zu finden, weil ohne Job kannst du auch keine Wohnung finanzieren, kannst du auch keine Wohnung haben ... " (Experte 4)

„Also wenn sie dann diesen Bescheid schon bekommen haben und es sich aussuchen ... eigentlich in der Stadt. Weil hier hat man mehr Möglichkeiten. Auf dem Land gibt es nichts. Also da sind ja auch wenige Afghanen ... zum Beispiel hier ... wir haben so viele Organisationen. ... wenn eine Familie herkommt, dann kann man ihm sagen: ,Bitte schicke deine Kinder in diese Schule oder jenes oder mach das. ' Wir haben zum Beispiel jetzt in unseren Vereinsbüros auch Deutschkurse, Englisch, Mathe für die Kinder von Flüchtlingen, die eigentlich das nicht leisten können, dass sie dann irgendwo eine Nachhilfe oder so was nehmen. ... Dass was wir eigentlich machen können, haben wir bis jetzt gemacht und werden wir auch in Zukunft machen. " (Experte 7)

„,Man hat ja alles in der Stadt, zusammen: den Sprachkurs, Arbeitsplatz, die Transportmittel und das Zusammenkommen und die Unterhaltungsmöglichkeiten von einem Lokaltreffen bis Disco bis Moschee, was sie auch immer ... Die meisten Hochzeiten werden in der Stadt gefeiert, weil hier eine Halle gemietet wird. ... 
Auf dem Land haben sie diese Möglichkeit nicht oder die Leute haben die Möglichkeit nicht, auf eine Hochzeit nach Wiener Neudorf zu fahren. Also, noch dazu ... die Stadt ist anonym. In der Stadt müssen sie die Leute nicht kennen und die Leute werden auch wenig ... gestört von den Migranten. ... Die meisten wollen in die Stadt gehen, weil es in der Stadt Arbeitsmöglichkeit gibt. Weil es in der Stadt schnelle Kommunikationsmöglichkeit gibt mit ihren eigenen Landsleuten, weil in der Stadt sie eine Wohnung finden können, leichter finden können. ... die meisten wollen nach Wien und das hat mehrere Hintergründe. Zum Beispiel die Jugendlichen sind als Flüchtlinge gekommen von Iran, Pakistan, da haben sie immer in den Städten gelebt. Und sie waren auf der Flucht immer in den Städten ...." (Experte 3)

„Das was die sagen, dass alle einen Kurs haben, das besuchen, das ist nicht wahr. In Wien funktioniert das. In Wien, jemand, der eigentlich will, dass er Deutsch lernt, ... das kann er. Es sind Mittel dazu da und die können das machen. Aber auf dem Land, das ist ein ganz kleiner Prozentsatz. " (Experte 7)

„Also die, die bei uns sind, die wollen eigentlich lieber in Wien sein. Und auch wenn sie irgendwo anders hin versetzt worden sind, war das eher eine Katastrophe. ... zweimal ist es so gewesen, dass dann Verlegungen ins weitere Niederösterreich oder ins Burgenland waren. ... aber sie wollten halt die Nähe zu Wien haben. Da haben sie auch doch wieder Freunde gehabt und das Netzwerk war halt da gegeben. Und das war anfänglich schon ein Problem “. (Experte 9)

„Ja, Wien ist sehr schön. Ich mag Wien. Ich war in Teheran und ich habe die Leute auch in Teheran gesehen, aber Wien ist die schönste. ... Ich war ein Monat weg. Ich war in Teheran, ... ich habe Wien vermisst, ... ich will einfach hier leben, solange ich lebe. " (Resp. 1, 170816)

„Ich bin nach Wien umgezogen, und es gefällt mir sehr hier. Ist eine große Stadt, mehr Menschen, ich habe viele Freunde und es wird mir nicht fad. " (Resp. 3, MT_170806)

“... in city, especially in capital you have more chances to develop yourself, to network and to meet people, to meet interesting people from around the world." (Resp 5, DF_170824)

„Ich fühle mich hier in Wien wohl, ... Ich habe mich an das Leben hier gewöhnt“" (Resp. 9, MT_170919)

„... ich bin zufrieden damit, aber es leben nicht so viele Österreicher/innen in der Umgebung, sonst viel mehr Migrant/innen, aber trotzdem ist es gut dort. Es gibt Parks in der Umgebung und wir gehen hin, und werden nicht sowie im Iran von den Menschen belästigt oder beleidigt. Die Infrastruktur der Stadt ist sehr gut, 
was den öffentlichen Transport betrifft. Ich habe sonst nirgendwo gelebt und war nur im dritten Bezirk. “ (Resp 8, MT_170919)

„Hier gibt es viele Leute und viele Geschäfte von Türkisch, Halal und so. ... von unserer Tradition und Kultur gibt es viel Möglichkeiten und viele Leute. Und viel Verkehr mit allen, das ist so gut. Aber wenn ich am Land wäre, ich bin mit dem Fahrrad gefahren und mein Leben war in Gefahr. Und deswegen ... ja, Stadt ist für mich sehr wichtig, und die Kinder können leicht zur Schule oder ins Gymnasium gehen und so leicht nach Hause kommen. Und man kann auch besser bis 10 oder 12 Uhr am Nacht spazieren gehen oder Eis ... aber am Land nicht, gibt es nicht diese Möglichkeit. “( Resp. 4/5, ML 170829)

„,Meiner Meinung nach ist das Leben in Wien leichter, ... Hier muss das Leben etwas leichter sein als am Land. ... Ich denke am Land wird es auch leicht sein, wenn man bessere Lebensmöglichkeiten hat wie Haus, Auto oder bestimmte Institutionen. Für mich wird es wohl schwer sein auf dem Land zu leben, da ich es ohne Auto nicht leicht haben werde. Ich werde nicht mobil sein. ... ich habe Freunde in Villach und in Linz. Ich habe regelmäßigen Kontakt zu diesen. Wenn ich diesen erzähle, dass ich an den Medizin-Veranstaltungen teilnehme, dann sagen sie, dass Wien viel besser ist als das Leben auf dem Land. “ (Resp. 1, WG_170807)

„Ich habe noch nicht wo anders gelebt, ich war nur in Linz, aber ich denke Wien ist besser, weil Menschen mit unterschiedlicher Herkunft wohnen, und weil hier die öffentlichen Verbindungen besser sind und Orte für Freizeitaktivitäten sind viele. Ich mag Wien sehr, und in Traiskirchen habe ich ihnen gesagt, dass ich nur nach Wien möchte. “ (Resp. 10, MT_170919)

„'I: Und wo ist es dir leichter gefallen Kontakte zu knüpfen? ... Also was fällt dir jetzt in Wien leichter als in Mödling?' B: ,Ja, genau. ' I: ,Und wie ist das in Wien für dich? Wo knüpfst du da die Kontakte? 'B: ,Überall. Überall wo ich will.' I: ,Aber wie funktioniert das? ' B: ,Es funktioniert einfach. ' I: ,Wie einfach? ' B: ,Ja, ich weiß nicht, wie ich es dir jetzt erklären. Aber es ist sicher einfach. Sicher einfacher als Mödling. ' I: ,Sind die Leute anders in Wien als in Mödling?' B: ,Nicht anders, aber ... Man geht mehr mit mehreren Leuten, kann mit verschiedenen Leuten reden. In der Stadt gibt es verschiedene Leute. ... in Wien gibt es aus verschiedenen Ländern und darum ist es einfacher. In Mödling habe ich fast alle gekannt. Weil meine Schule war in Mödling und Mödling ist auch klein. ... Aber hier in Wien sieht man jede Sekunde irgendwie einen anderen Menschen. ... Ich habe Freund aus Russland, aus Ägypten, aus Türkei, aus Bosnien, aus Serbien, aus Ungarn, aus Deutschland, aus Brasilien sogar, Afghanistan oder Indien, Pakistan. Ja, aus Österreich sogar. Ich habe verschiedene Freunde. ... ' I: ,Ist das also auch ein Grund, warum du in Wien bleiben willst? 'B: ,Ja, genau!' (Resp. 1, ML_170816) 
„Nachdem ich meinen Bescheid bekommen habe, bin ich in Österreich herumgereist, von Salzburg über Tirol, Niederösterreich und andere Bundesländer. Wien ist für mich eine sehr ruhige Stadt und ich fühle mich hier wohl. Es kann sein, dass ich mich manchmal alleine fühle. Es liegt daran, dass ich hier nicht viele Bekannte oder Freunde habe, aber ich habe hier eine österreichische Familie, die ich als meine Familie bezeichne. Der größte Wert, der mir entgegengebracht wird hier, in dieser Stadt, dass man mich als Mensch wahrnimmt und mich respektiert, und wenn du Ziele hast und dafür arbeitest, erreichst du sie auch." (Resp. 2, MT_170731)

„,Wien hat viele Möglichkeiten. Es ist eine kleine Metropole. Also dafür, dass Wien echt so klein ist, hat es echt viel zu bieten. Man kann hier problemlos zur Schule. Also Wien ist einfach so die kleine perfekte Stadt zum Leben für Familien, für mich persönlich auch. Also ich würde gerne selber wegziehen, aber wenn ich meine Familie gründen möchte, würde ich gerne dann nach Wien zurück, weil man hat echt alles hier. “ (Resp. 14, VR_170828)

„... Es war schwer, wirklich. Es war schwer [Anm.: Gemeint ist das Knüpfen sozialer Kontakte] ,I: Warum war es schwer? 'B: Weil man muss ein Auto haben, damit man Freunde treffen kann oder so. Oder keine Ahnung, irgendwo hinfahren. Weil es gibt keine öffentlichen Fahrzeuge, dass man hinkommt. Und da es so was nicht gibt, dann Kontakt zu knüpfen ist auch nicht so schön. “(Resp. 1, ML_170816)

„, Also in einer großen Stadt hat man mehr Ausgaben als in einer kleineren Stadt. Man verdient aber wiederum in der Großstadt wesentlich mehr als am Land. Deswegen bin ich der Meinung, dass es überhaupt keine Rolle spielt, ob man in einer Stadt, am Land, oder in einer Großstadt wie Wien lebt. Die Möglichkeiten, die du in Wien hast, hast du auch in Eisenstadt oder St. Pölten. Einen besonderen Vorteil, den man allerdings in einer Großstadt hat, sind natürlich die öffentlichen Verkehrsmittel. " (Resp. 2, JN 170816)

\subsection{Plädoyers für die Integration in ländlichen Gemeinden}

Ungeachtet einer deutlichen Dominanz der Argumente für die Binnenmigration nach Wien hoben einige Befragte auch vielfältige Positiva des Lebens in Kleinstädten oder ländlichen Kleingemeinden hervor. Diese bezogen sich vor allem auf die einfacheren Möglichkeiten der Aufnahme sozialer Kontakte etwa mit den Menschen in der Wohnnachbarschaft sowie auf Umwelt- und Lebensqualitätsaspekte. Hinsichtlich der strukturellen Integration wurde u.a. der Zugang zu Sprachkursen als Positivum angeführt, aber bezogen auf die Arbeits- und Wohnungsmarktintegration kaum Vorteile genannt: 
„ 'I: Wieso haben Sie sich entschieden hier zu wohnen, und nicht in Wien? ' B: ,Wie wir in dem Heim gewohnt haben, hatten wir Kontakt zu zwei arabischen Familien, sie haben dann hier eine Wohnung gefunden. Sie haben uns dann gesagt, dass es hier sehr gut ist und ruhig. Man kann auch schneller und leichter einen Sprachkurs finden. Ich war auch schwanger und dachte, dass ich hier dann auch in den Sprachkurs gehen kann mit meinem Kind. In Wien gibt es nicht so viele Möglichkeiten. Das Kind muss drei Jahre alt sein, damit man in den Sprachkurs gehen darf." (Resp. 13, ML_171102)

„,Ich weiß nicht, aber hier ist es so: Du wohnst seit zehn Jahren in einer Wohnung und $d u$ weißt nicht, ob die Nachbarin, die rechts von dir wohnt, gerade da ist oder nicht. Ich würde nicht sagen, dass die Menschen nicht kontaktfreudig oder sozial sind, aber es ist einfach so. Hier hat man sich daran gewöhnt. ... ich kann mich als Kind erinnern, ... und dort zum Beispiel haben wir ein Haus am Anfang der Straße gehabt und ich wusste bis zum allerletzten Haus der Straße, wem das Haus gehört. Also die Menschen dort sind einfach viel kontaktfreudiger. Sozialer würde ich sagen. Vielleicht hängt es auch damit zusammen, dass die Frauen nicht arbeiten, dass die Aufgaben der Frauen Kindererziehung und Haushalt sind. Vielleicht haben sie dadurch viel mehr Zeit, weil sie nicht arbeiten müssen. " (Resp. 10, VR_170808)

„,Aber ein Dorf ist klein und grün und dort wohnen weniger Menschen und es ist leichter Bekanntschaften mit den Menschen zu machen. ... Im Sprachkurs kannte ich viele Menschen. Die Frau Patricia seitens des Staats hat uns sehr viel geholfen. Ich habe auch eine sehr nette Nachbarin und meine Nachbarn sind alle Österreicher/innen und sind alle sehr nette Menschen. “(Resp. 14, ML_171102)

„Ich liebe Landleben auf jeden Fall. Ich steh` auf Berge, auf Wasserfälle, auf Skifahren auf jeden Fall. Aber das ist wieder diese afghanische Einstellung: Ich kann nicht nach Tirol ziehen, weil meine Eltern alt geworden sind; die sind in Wien. Da will ich bei denen so, bei denen bleiben. “(Resp. 12, ML_171003)

\subsection{Manifestationen der Vulnerabilität im ländlich-kleinstädtischen Raum und deren Auswirkungen auf Binnenmigrationsent- scheidungen}

In den Interviews wurden von den Geflüchteten unterschiedlichste Aspekte der Vulnerabilität angesprochen, die ihren Aufenthalt vor allem in ländlichen Gemeinden bzw. kleineren Städten belasteten und letztlich die Entscheidung für eine Binnenmigration nach Wien maßgeblich beeinflusst haben. Diese bezogen sich sowohl auf die „determinants of vulnerability“ als auch auf deren „manifestations“, vor allem in der Subkategorie der „,intensified“, aber auch der durch bürokratische Systeme bedingten 
(vgl. Sabates-Wheeler \& Waite 2003, 13 und Abb. 1), so z.B. die Unkenntnis der räumlichen und regionalen Gegebenheiten sowie Gepflogenheiten, Diskriminierung im Zugang zu wichtigen Serviceleistungen (z.B. Sprachkurse), mangelndes Verständnis der lokal gängigen Sprache (regionale Dialekte!), soziale Diskriminierung bis hin zu expliziten Xenophobieerfahrungen und gesundheitliche Beeinträchtigungen. Bei Letzteren wurden von unseren Respondent/inn/en ausschließlich psychische Probleme wie Depressionen infolge sozialer Isolation oder Diskriminierung genannt:

„... Und es gibt auch andere Familien, die sind in einem Asylheim, die haben keine Möglichkeit Deutsch zu lernen. Die [österreichische] Bevölkerung hatte vor diesen Personen Angst, die dort wohnen. “ (Experte 2)

„Das Problem ist, das Leben auf Land ist für sie nicht attraktiv, sondern langweilig. Sie werden irgendwo auf einen Berg, in ein Tal, da gibt es kein Einkaufszentrum, also für die noch nicht anerkannten, keinen Sprachkurs und dort, schon von Anfang an, haben sie eine Aversion dann gegen das Land und sie wollen in die Stadt ziehen. ... Auf dem Land, wenn sie in einem Dorf sehen, Flüchtlinge gehen dorthin irgendwo. In einer Pension, wenn sie dort herumgehen, fallen sie sofort auf. ... Und auf dem Land gibt es auch keine Arbeit, keine Wohnmöglichkeiten. Wenn sie jetzt von der Pension rausziehen, ein Zimmer mieten wollen, da gibt es kaum ein Zimmer dort zu mieten. Dann müssen sie in die Stadt gehen. “ (Experte 3)

„Montagabend war ich bei einer afghanischen Hochzeit, und da habe ich einen Herrn getroffen, der bei Rohrbach gelebt hat. ... Und es war die einzige Familie dort in diesem Dorf. ... Deswegen waren sie die Superstars, also alle kannten die Familie. ... Die waren so berühmt dort. ... Also alle waren glücklich bis auf die Frau, weil die Frau sich dort alleine gefühlt hat. Und als sie nach Wien gekommen sind, jetzt sind sie plötzlich alle glücklich. Warum? Weil sie in Wien sind." (Experte 6)

„In Leoben geht gar nicht. ... Ist in Wien leichter. Sicher! In Leoben geht es nur in diesem Fall, wenn die Leute zu dir kommen. Du hast aber keine große Chance zu jemandem zu gehen: ,Hey, servus!' ... Aber Wien auf jeden Fall [ist es] irgendwie besser. Weil die Leute, ... die behandeln dich besser und die antworten dir. Aber in einem Dorf, die dürfen auch nicht antworten. Weil es einfach ein Dorf ist. " (Resp. 3, 170710)

„' 'Ich fühle mich hier in Wien wohl, ... Ich habe mich an das Leben hier gewöhnt." I: ,In welchen Orten war es für Sie schwierig Kontakte zu knüpfen?' B: ,In Vorarlberg, weil die Menschen dort eine andere Aussprache haben. Mir fällt es schwer sie zu verstehen. Einmal musste ich dort zur Krankenkasse gehen und mein E-Card aktualisieren. Die Angestellten waren nicht freundlich mir gegenüber. "“ (Resp. 9, MT_170919) 
„Ich habe in Wiener Neustadt in einem kleinen Dorf gewohnt, in dem nur alte Menschen wohnten. Die Jungen sind in die Stadt zum Arbeit und Studieren umgezogen. Der Ort war überhaupt nicht gut für meine Psyche. " (Resp. 3, 170806)

„'I: Und wieso nach Wien?' B: ,Weil damals war ich allein, und ich wollte in der Stadt leben, wegen meinem Kind, ... und die beiden waren ganz klein. Ja, es war so fad für mich, obwohl es sehr nette Leute waren, sehr schöne Stadt, aber trotzdem. Und ich bin aufgewachsen in einer Stadt, in Kabul. Deswegen ich wollte nach Wien kommen, ja. ' I: ,Hatten Sie auch das Gefühl, dass die Leute Ihnen hier mehr helfen oder ...?' B: ,Ja, genau, je genau. Das habe ich auch. Deswegen bin ich auch gekommen. Weil bin ich alleine gewesen und ohne Mann und mit zwei Kindern, das ist sehr schwer, ja. “" (Resp. 6, DF 170825)

\subsection{Die Lokalisation der ethnischen Community als Determinante räumlicher Mobilitätsentscheidungen}

In der Diskussion um Wohnsegregation wird nicht selten die Formation von sogenannten Parallelgesellschaften als Gefahr für die soziale Kohäsion der urbanen Gesellschaft (vgl. Augustin 2012; Manea et al. 2017) angesprochen. Dieses Argument hat aus integrationspolitischer Perspektive einerseits eine gewisse Berechtigung, es sollte aber andererseits nicht übersehen werden, welche wichtige Rolle die ethnische Community auch im Rahmen der sozialen und strukturellen Integration auf dem Arbeitsund Wohnungsmarkt spielt (vgl. u.a. DAMm 2009a; DANZER \& YAMAn 2013). Es wurde daher in der Befragung auch explizit nachgefragt, inwieweit die Lokalisation einer ethnischen Community aus Afghanistan eine Relevanz für die Wohnortentscheidung besitzt, um abzuschätzen, inwieweit Tendenzen einer lokalen „community formation“ für die rezent zugewanderten Geflüchteten aus Afghanistan wichtig sind. Die Palette an Meinungen dazu ist widersprüchlich, es dominiert die Einstellung, dass eine konfliktfreie interethnische Koexistenz mit allen hier lebenden Gruppen angestrebt wird und die ethnische Zusammensetzung der Bevölkerung des gewählten Wohnorts, v.a. aber der lokalen Nachbarschaft, weitgehend gleichgültig ist.

Die afghanische Community wird zwar in Bezug auf die Infrastruktur und bestimmte Hilfestellungen positiv bewertet, aber nicht ausschließlich, da auch soziale Kontrolle und Einschränkungen der persönlichen Freiheit damit einhergehen. Das Risiko einer gewissen „Abschottung“ gegenüber der Aufnahmegesellschaft wird von einigen Interviewten realistisch eingeschätzt, wobei u.a. das Erlernen der deutschen Sprache erschwert wird. Als Resümee aus den Einschätzungen der befragten Afghan/ inn/en sowie aus den Expertenaussagen ist ein deutliches Bewusstsein für die Wichtigkeit sowohl von sozialen Kontakten innerhalb der eigenen ethnischen Community als auch mit der Mehrheitsgesellschaft abzuleiten. 
Nach Einschätzung der Expert/inn/en spielt die ethnische Community im Alltagsleben, für soziale Interaktionen sowie als Integrationshilfe eine erhebliche Rolle, woraus abzuleiten ist, dass die ausschließlich in den großen Städten Österreichs und vor allem in Wien lokalisierte afghanische Community sehr wohl eine mobilitätsstimulierende Anziehungskraft in Richtung auf die Metropole ausübt:

„Viele wandern dann aus den Bundesländern selbständig nach Wien, weil entweder denken sie, dieser Bezug zu den Communities ist da ... “ (Expertin 8)

„Ich glaube diese Möglichkeit, also Stadt, Dorf, je nachdem. Das ist eigentlich für die Integration denke ich, dass es gut ist, wenn man mit den Einheimischen viel Kontakt hat und viel zu tun hat als nur in der Gesellschaft in der eigenen Community ... Ich persönlich meine, es ist beides wichtig, sowohl der Kontakt zu der Aufnahmegesellschaft, aber auch Kontakt zu der eigenen Community, weil hier braucht man auch ein bisschen soziale Kontakte ... Kontakte aus der Heimat. Auch für die Anerkennung, weil dann weiß man: ,Ok, ich bin nicht hier alleine, sondern es gibt auch andere, die was gemacht haben. 'Aber auch als Rollenbild, ,role models', ... bin neu, Flüchtling, komme nach Wien, dann plötzlich treffe ich einen Afghanen, der bei diesem Amt oder dort als Arzt arbeitet und so. Dann plötzlich werde ich auch motiviert und sage: ,Ok, was hast du gemacht? Und wie hast du es geschafft?' Deswegen ist beides aus meiner Sicht wichtig. " (Experte 6)

Im Gegensatz dazu bewerten die afghanischen Respondent/inn/en die Existenz der ethnischen Diaspora generell sowie die Möglichkeit des Zusammenlebens mit Landsleuten im räumlichen Kontext des Wohnviertels durchwegs als viel weniger relevant:

„Aber im Ort, wo ich lebe, mag ich, dass keine Afghanen leben, ... Weil, egal ob Österreicher oder Afghane, sind sowohl gut oder schlecht. Und in meinen Wohnort ist es mir wichtig, was die Österreicher/innen darüber denken und wenn es positiv ist, würde ich mit ihnen wohnen, und wenn nicht, dann würde ich mich fernhalten. “(Resp. 10, MT_170919)

„Es ist mir gleichgültig, ob es Afghanen oder Österreicher gibt. Wenn man selber nicht problematisch ist, dann hat man auch keine Probleme mit dem Leben. Die Gegend und die Menschen herum spielen keine Rolle, wenn man das Leben selber gut bewältigen kann. Hauptsache ist, dass man selber ein guter Mensch ist, dann kann man überall sehr gut auskommen, dabei spielt es keine Rolle, ob im Umfeld Afghanen oder Österreicher leben. Aber es gibt Menschen in der Gesellschaft, die sehr problematisch sind. Diese Menschen sind dann sowohl in der Gesellschaft als auch für den Staat sehr auffällig. Mit diesen möchte ich nicht zusammen in einer Gegend leben. “(Resp. 1, WG_170807)

„Nein, so wichtig ist es auch nicht. Es ist mir egal, ob Österreicher/innen oder andere Nationalitäten bei uns wohnen. Wichtig ist, dass es Menschen sind. Sie 
müssen Mensch sein und Menschlichkeit zeigen und miteinander leben und das Leben genießen. " (Resp. 4, WG_170820)

„Es macht mir überhaupt nichts aus, wenn da andere Nationalitäten leben. Wichtig ist, dass sie ruhig sind und sich niemand in andere Angelegenheiten einmischt. Jede/r sollte sich um ihre/seine Sache kümmern. “ (Resp. 5, MT_170814)

„I: ,Das heißt, es war für dich nicht wichtig, dass der Wohnort wo ist, wo du Kontakte haben könntest. 'B: ,Nein! Und jetzt habe ich so einen großen Kreis von Verwandten und ... Außer meiner Familie, ja. Weit entfernte Verwandte von meinem Vater und meiner Mutter. "“ (Resp. 13, VR_170821)

„,Meinst du, wo ich wohne, ... müssen Afghanen sein? ...Nein, das war für mich nicht wichtig ... Soll ich dir Grund sagen? ... Ich wohne nicht in Afghanistan, ich wohne in Österreich, ich muss das zugeben. Ich brauche die anderen Dinge, die ich lernen kann. Und wenn ich z.B. jeden Tag 24 Stunden mit Afghanen unterwegs bin, wo kann ich Deutsch lernen? Wo kann ich die Kultur, von wo soll ich das wissen, was es gibt? Und deswegen das war für mich nicht so wichtig. Natürlich das ist wichtig, z.B. für Kontakt. Man kann auch nicht ohne Landsleute sofort leben. Aber ich habe am Anfang mehr als genug Kontakt gehabt von diesem Kulturverein. “(Resp. 8, VR_170802)

Dass integrationsorientierte Familien sogar Wohnortwechsel vollziehen, um nicht in einem Umfeld zu leben, in welchem die afghanische Community zahlreich lebt, belegt das folgende Statement einer bereits länger in Wien ansässigen Afghanin. Der Umzug in einen anderen Wiener Bezirk wurde im Sinne der Kinder getätigt, um ihnen den Erwerb der deutschen Sprache durch den Freizeitgebrauch zu erleichtern. Dies war in ihrem vormaligen nachbarschaftlichen Umfeld mit fast ausschließlich afghanischen Freund/inn/en kaum möglich.

„, ,Und warum seid ihr aus dem 11. Bezirk weggezogen? Also die größere Wohnung hättet ihr auch dort haben können, wo alle Familien sind. 'B: ,Weil es war besser, dass wir weggekommen sind, ... vom 11. Bezirk, weil da waren sehr viele afghanische Familien. Und wenn die Kinder alle draußen waren, haben sie nur in ihrer Sprache geredet. So war es für uns besser. ... Ich glaube, dadurch haben wir dann schneller Deutsch gelernt. Wir mussten dann reden. “ (Resp. 15, VR_170829) 


\subsection{Die Bedarfsorientierte Mindestsicherung (BMS) - ein Kausalfaktor der Binnenmigration?}

In der politischen und medialen Diskussion ${ }^{8}$ der vergangenen Jahre wurde häufig ein Konnex zwischen der unterschiedlichen Höhe der Mindestsicherung und einer nach Wien orientierten Binnenmigration nach Erhalt des Asylbescheids hergestellt. Trotz expliziter Nachfragen in den Interviews, wurden hierzu seitens der Respondent/inn/en wie auch der Expert/inn/en nur wenige Statements abgegeben. Die daraus ableitbaren Schlussfolgerungen sind also sehr eingeschränkt, denn einerseits wird der Konnex zwischen der Höhe der Bedarfsorientierten Mindestsicherung und der nach Wien orientierten Migration bestätigt sowie andererseits als nicht relevant eingestuft. Die BMS hilft vor allem den besonders vulnerablen Subgruppen unter den Geflüchteten, also Älteren mit geringen Erwerbschancen, alleinerziehenden Frauen, aus gesundheitlichen Gründen schwer Vermittelbaren, jenen mit geringen Bildungsniveaus sowie generell allen bis zum Finden einer Erwerbsarbeit, ihren Lebensunterhalt zu bestreiten. Für etliche dieser Gruppen sind allerdings auch eher beschränkte Möglichkeiten der Binnenmobilität anzunehmen. Aufgrund der in den Interviews an anderen Stellen geäußerten durchwegs hohen Erwerbsmotivation (vor allem auch der weiblichen Befragten) ist jedoch anzunehmen, dass nur in den seltensten Fällen die Höhe bundeslandspezifischer finanzieller Transferleistungen den allein ausschlaggebenden Faktor für Binnenmigration darstellt. Häufiger wird eine Kombination von verschiedensten Faktoren eine Rolle spielen. Darunter fallen z.B. familiäre soziale Netzwerke, strukturelle Ressourcen der ethnischen Community, Erwerbschancen, Gegebenheiten auf dem Wohnungsmarkt usw. Es handelt sich also vielfach um jene Faktoren, welche im Kontext der Argumentation für eine Binnenmigration in eine urbane Agglomeration ganz allgemein bereits ins Treffen geführt wurden.

Für die Relevanz der Mindestsicherung als Kausalfaktor einer nach Wien orientierten Binnenmigration spricht:

„, 'Und denken Sie, dass diese bundeslandspezifischen Sozialleistungen da auch eine Rolle spielen?' R: ,Ja, das sicherlich, ja, hundertprozentig. Nur, dass wird dann jetzt glaub' ich bald eh eingeschränkt. Das ist schon eingeschränkt, da können sie nicht leicht hierherkommen. Nur in Wien ist auch jetzt,, dieses Problem, was Wohnen betrifft schwierig. Wenn jemand keine Wohnung hier findet, wie soll er denn dann da daherkommen? Das ist dann schon ein Problem. "“ (Experte 7)

Gegen die Mindestsicherung als primärer Kausalfaktor einer nach Wien orientierten Binnenmigration und für ein Zusammenwirken verschiedenster Faktoren sprechen folgende Zitate:

8 Vgl. dazu <https://diepresse.com/home/panorama/wien/5229335/Mindestsicherung_Werzieht-des-Geldes-wegen-nach-Wien>; $\quad<$ https://derstandard.at/2000042670101/Mindestsicherung-zieht-Asylberechtigte-verstaerkt-nach-Wien>. 
„Und die bleiben nicht in anderen Städten, Orten und die werden nach Wien kommen, in andere Bundesländer, Städte kommen. Es ist nicht die Schuld von den Afghan/inn/en. Viele Österreicher/innen sagen: ,Es ist nur wegen dem Sozialgeld!' Das stimmt auch nicht meiner Meinung nach. Es ist [dass sie in die Städte gehen], weil sie nicht von der Ortschaft aufgenommen wurden, nicht akzeptiert wurden, nicht in diese Ortschaft zumindest integriert wurden. " (Experte 2)

„.... Es ist egal, dass das Zuhause weit weg ist von Salzburg, wo ich arbeiten gehe. Das ist mir wurscht, aber Hauptsache meine Familie und ich haben dort ein Zuhause gefunden, andere Bekannte gefunden und der wird nie nach Wien kommen. Und obwohl die Mindestsicherung in Salzburg ganz, ganz wenig ist." (Experte 2)

„Ihre Rolle ist sehr wichtig, aber auch nicht so viel. Aber man ist doch auf diese Unterstützung angewiesen, um die Miete zu zahlen und ihr Leben zu finanzieren, solang man nicht arbeiten darf, oder keinen Job hat. “ (Resp. 1, MT_170711)

\section{Conclusio}

Im Fokus stand die Beantwortung der Fragen, was die Attraktivität der Metropolen für die Binnenmigration von Geflüchteten Afghan/inn/en ausmacht, inwieweit auch Vulnerabilität die Binnenmigration in die urbanen Zentren beeinflusst und welche weiteren Faktoren für die Mobilitätsentscheidung, d.h. den Wohnsitzwechsel in eine urbane Agglomeration, eine Rolle spielen?

Faktum ist, dass die Vorteile der Stadt vielfältig sind und seitens der Befragten auch aus individueller Perspektive sehr divergent bewertet werden. $\mathrm{Zu}$ den von den Interviewten aufgezählten Positiva der Stadt Wien zählt die generalisierte Erwartung, dass vieles in Wien leichter fallen könnte. Dabei spielen die Faktoren der strukturellen Integration, also Arbeitsplatz und Wohnmöglichkeit, eine primäre Rolle. Dazu tritt aber eine große Zahl weiterer Determinanten: bestehende soziale Netze zu Verwandten und Freunden, die Einbindung in großfamiliäre Strukturen, Vorhandensein einer ethnischen Community, religiöse Infrastruktur, das Angebot des öffentlichen Personennahverkehrs, Zugang zu Bildung und beruflicher Weiterbildung, Deutschkurse, Unterhaltungs- und Freizeitangebote, Wellbeing allgemein, die Erwartung des Nichtvorhandenseins von Xenophobie, die Gewohnheit des Lebens im urbanen Kontext im Herkunftsland, das Fehlen von Meeting Places im öffentlichen Raum in Kleingemeinden, die Anonymität etc. Unterschiedliche Levels der Kontaktfreudigkeit der lokalen Bevölkerung sowie die Möglichkeiten der spontanen Kontaktaufnahme auch mit unbekannten Menschen werden als weitere Pluspunkte der Großstadt angeführt. Eine spezielle Barriere bilden lokale dialektale Besonderheiten, die es den Geflüchteten erschweren, sprachlich leich- 
ter Fuß zu fassen. Soziale Isolation wird je nach individueller Wahrnehmung und Erfahrung ins Treffen geführt, ebenso daraus resultierende psychische Probleme. Die meisten Befragten äußern sich sehr klar dazu, wo sie dauerhaft lieber leben möchten und so heben einige auch Vorteile von kleinstädtischen bzw. ruralen Siedlungen hervor.

Aus dem empirischen Material lässt sich bei den meisten Geflüchteten eine nicht quantifizierbare Präferenz für das Leben im urbanen Kontext sowie daraus resultierend ein Trend zur faktischen sowie beabsichtigten Binnenmigration der Asylberechtigten nach Wien herauslesen. Diese ist allerdings häufig als Ausdruck großer und oftmals unrealistischer Hoffnungen zu sehen und weniger als eine realitätsgetreue Einschätzung der tatsächlichen Lage. Ungeachtet der prekären Situation auf dem Wohnungsmarkt besteht also ein Trend der Mobilität in die urbanen Ballungsräume. Es existiert die Hoffnung, in der Stadt rasch eine attraktive Wohnung zu finden, das Gegenteil ist in der Regel der Fall. Die Gründe für diese stadtzentrierte Migration sind, wie unsere Befragung ergeben hat, individuell sehr unterschiedlich. Vulnerabilitätsbezogene Faktoren spielen aber offensichtlich eine Rolle. Zugleich muss betont werden, dass für keine/-n einzige/-n Befragte/-n die in den Bundesländern unterschiedlichen Bezugsgrößen der Mindestsicherung der allein ausschlaggebende Faktor für eine Wohnsitzverlegung waren, jedoch eines unter diversen Argumenten für den Wegzug nach Wien. Die Lokalisation der afghanischen Community sowie deren Infrastruktur ist sehr wohl relevant, aber nicht im Sinne eines Wunsches nach klein- oder großräumiger ethnischer Wohnsegregation im Stadtgebiet. Die Geflüchteten sind durchwegs nicht daran interessiert, in unmittelbarer Nachbarschaft mit Landsleuten zu wohnen, die Infrastruktur der Community, die aber primär in Wien verfügbar ist, wird aber sehr wohl als relevant bewertet.

\section{Bibliographie}

Abrahamsson M., Borgegård L.E. and Fransson U. (2002), Housing Careers: Immigrants in Local Swedish Housing Markets. Housing Studies, 17 (3), pp. 445-464.

AdDendum (28-04-2018). Der Flüchtling als Mieter. <https://www.addendum.org/wohnen/derfluechtling-als-mieter/> [Zugriff 28-01-2020]

Aldén L., and Hammarstedt M. (2014), Integration of immigrants on the Swedish labour market: recent trends explanations (Linnaeus University Centre for Labour Market and Discrimination Studies, No. 2014, 9).

Altemeyer-Bartscher M., Holtemöller O., Lindner A., Schmalzbauer A. and Zeddies G. (2016), On the Distribution of Refugees in the EU, Intereconomics 51 (2), 220-228. $<$ https://archive.intereconomics.eu/year/2016/4/on-the-distribution-of-refugees-in-the$\mathrm{eu} />$ [Zugriff 28-01-2020].

Andersson R. (2003), Settlement dispersal of immigrants and refugees inEurope: Policy and outcomes. Vancouver, Vancouver Centre of Excellence. 
Andersson R. and MekKonen T. (1996), The Geographical and socialmobility of immigrants. The impacts of the whole of Sweden Strategy, Geografiska Annaler. Series B, Human Geography, 78, pp. 3-25.

ANDERSSON R., BRÅmÅ Å. and HolmQvist E. (2010), Counteracting segregation: Swedish policies and experiences. Housing Studies, 25(2), pp. 237-356.

ÅsLund O. (2005), Now and forever? Initial and subsequent location choices of immigrants. Regional Science and Urban Economics, 35 (2), pp. 141-165.

AsylKoordination (ed.) (2020), Mangelberufe <https://www.migration.gv.at/de/formen-derzuwanderung/dauerhafte-zuwanderung/bundesweite-mangelberufe/> sowie $<\mathrm{https} / /$ www.migration.gv.at/de/formen-der-zuwanderung/dauerhafte-zuwanderung/regionalemangelberufe/> [Zugriff 26-01-2020].

Augustin J. (2012), Das Phänomen der Parallelgesellschaft. Am Beispiel Ottakring. Diplomarbeit, Universität Wien. Fakultät für Sozialwissenschaften $<$ http://othes.univie.ac. at/18099/1/2012-01-25_0400406.pdf > [Zugriff 28-01-2020].

European Migration Network (ed.) (2017), Asylum shopping. In: EMN Glossary. European Commission, Migration and Home Affairs <https://ec.europa.eu/home-affairs/ content/ asylum-shopping_en $>$ [accessed 01-12-2019].

Birgier D.P., Haberfeld Y., Lundh C. and Elldér E. (2016), Selectivity and internal migration: A study of refugees' dispersal policy in Sweden, $18^{\text {th }}$ Nordic Conference contribution, Migration Conference - "Migration and social inequality: Global perspectives - new boundaries”. 11-12 August 2016 Oslo, Norway.

Bloem J. and Loveridge S. (2017), The secondary migration of refugees resettled in the US. Forced Migration Review, 54, pp. 26-28.

Bloem J. and Loveridge S. (2018), The Costs of Secondary Migration: Perspectives from Local Voluntary Agencies in the USA. Journal of International Migration and Integration, 19 (2), 233-251.

Boswell C. (2003), Burden-sharing in the European Union: lessons from the German and UK experience, Journal of Refugee Studies, 16, pp. 316-335.

BrekKe J.P. and AARSET M.F. (2009), Why Norway? Understanding Asylum Destinations. Oslo: Institute for Social Research, <https://www.udi.no/globalassets/global/ forskning-fou_i/ beskyttelse/why-norway.pdf $>$ [accessed 06-11-2019].

BUNDESMINISTERIUM FÜR INNERES (BM.I) (2016), Asylstatistik 2015 <https://www.bmi.gv.at/301/ Statistiken/files/Jahresstatistiken/Asyl_Jahresstatistik_2015.pdf> [Zugriff 31-10-2019].

BUNDESMINISTERIUM FÜR INNERES (BM.I) (2017), Asylstatistik $2016<$ https://www.bmi.gv.at/301/ Statistiken/files/Jahresstatistiken/Asyl_Jahresstatistik_2016.pdf $>$ [Zugriff 22-01-2020].

BUNDESMINISTERIUM FÜR INNERES (BM.I) (2018), Asylstatistik $2017<$ https://www.bmi.gv.at/301/ Statistiken/files/Jahresstatistiken/Asyl-Jahresstatistik_2018.pdf> [Zugriff 21-01-2020].

BUNDESMINISTERIUM FÜR INNERES (BM.I) (2018), Niederlassungs- und Aufenthaltsstatistik. November $2018<$ https://www.bmi.gv.at/302/Statistik/files/2018/Niederlassungs-_und_ Aufenthaltsstatistik_November_2018.pdf $>$ [Zugriff 21-01-2020].

BUNDESMINISTERIUM FÜR INNERES (BM.I) (2019a), Asylstatistik $2018<$ https://www.bmi.gv.at/301/ Statistiken/files/Jahresstatistiken/Asyl-Jahresstatistik_2019.pdf $>$ [Zugriff 21-01-2020].

BUNDESMINISTERIUM FÜR INNERES (BMI) (2019b), Grundversorgung. Unterbringung und Betreuung $<$ https://www.bmi.gv.at/303/start.aspx $>$ [Zugriff 28-02-2019]. 
Dahlberg M. and Valeyatheepillay M. (2018), On the Anatomy of a Refugee Dispersal Policy: Neighborhood Integration and Dynamic Sorting, ifo Working Paper No. 285 [https://www. cesifo-group.de/DocDL/wp-2018-285-dahlberg-valeyatheepillay-refugee-integration.pdf; Zugriff 21-01-2020].

Damm A.P. (2009a), Ethnic Enclaves and Immigrant Labor Market Outcomes: Quasi-Experimental Evidence. Journal of Labor Economics, 27 (2), pp. 281-314 <https://cream-migration.org/ files/enclaveff16.pdf $>$ [Zugriff 29-01-2020].

Dамм A.P. (2009b), Determinants of recent immigrants' location choices: Quasi-experimental evidence. Journal of Population Economics, 22 (1), pp. 145-174. <https://www.econ. $\mathrm{ku} . \mathrm{dk} / \mathrm{cam} / \mathrm{wp} 0910 / \mathrm{wp} 0406 / 2005-17 . p d f>$ [Zugriff 03-02-2020].

Damm P.A. and Rosholm M. (2005), Employment Effects of Spatial Dispersal of Refugees - CAM (Centre for Applied Microeconometrics Institute of Economics University of Copenhagen) $<$ https://www.econ.ku.dk/cam/wp0910/wp0406/2005-03.pdf> [accessed 27-02-2019].

DANZer A.M. and Yaman F. (2013), Do ethnic enclaves impede immigrants' integration? Evidence from a quasi-experimental social-interaction approach, Review of International Economics, 21, pp. 311-325.

DARLING J. (2016), Privatising asylum: neoliberalisation, depoliticisation and the governance of forced migration $<$ https://doi.org/10.1111/tran.12118>, <https://rgs-ibg.onlinelibrary.wiley. com/doi/full/10.1111/tran.12118> [Zugriff 23-01-2020].

Diakonie FlüChtlingSDienst (ed.) (o.J.), Informationsblatt für Klient*innen zur Wohnsitzbeschränkung <https://www.asyl.at/aduploads/141.04.ma,wohnsitzbeschrnkungdeutsch. pdf $>$ [Zugriff 26-01-2020].

EuroCities (ed.) (March 2016), Social Affairs. Refugee Reception and Integration in Cities. $<$ https://ec.europa.eu/futurium/sites/futurium/files/eurocities-refugees-report.pdf $>$ [Zugriff 26-01-2020].

EXPERTENRAT FÜR INTEGRATION (ed.) (2015), 50-Punkte-Plan zur Integration von Asylberechtigten und subsidiär Schutzberechtigten in Österreich <https://www.bmeia.gv.at/fileadmin/ user_upload/Zentrale/Integration/Nationale_Integrationsfoerderung/50_Punkte-Plan.pdf $>$ [Zugriff 27-02-2019].

FABRY C. (19-07-2018), Verteilung der Flüchtlinge stark Wien-lastig, Die Presse <https:// diepresse.com/home/innenpolitik/5466711/Verteilung-der-Fluechtlinge-stark-Wienlastig > [Zugriff 28-11-2019].

Fratesi U., Percoco M. and Proietti P. (2014), Who welcomes them? The spatial distribution of refugees in Italy between attitude and opportunity $<\mathrm{https}: / /$ marcopercoco.files.wordpress. com/2014/11/rifugiati_final.pdf $>$ [accessed 02-12-2019].

Grimm O. and Bastaroli S. (21-06-2018), Wie verhindert man Asylshopping, Die Presse $<$ https:// www.diepresse.com/5451226/wie-verhindert-man-asylshopping $>$ [Zugriff 22-01-2020].

HAJEK P. (2017), Motivationen für österreichische Binnenmigration von Asyl- und subsidiär Schutzberechtigten nach Wien. Forschungsbericht <https://www.bmeia.gv.at/fileadmin/ user_upload/Zentrale/Integration/Studien/Studie_Binnenmigration_FINAL.pdf> [Zugriff 03-12-2019].

Hoekstra M., Kohlbacher J. and Rauhut D. (2018), Migration governance in European cities: What are the possibilities for local governments to create inclusive societies? In: LACROIX T. and Desille A. (eds.), Current trends and realities in urban migration governance. London and New York, Palgrave Macmillan, pp.17-38 < doi: 10.1007/978-3-319-65996$12>$. 
Hynes P. (2011), The dispersal and social exclusion of asylum seekers: between liminality and belonging. Bristol, Bristol University Press.

Kelly M. and Hedman L. (2016), Between Opportunity and Constraint: Understanding the Onward Migration of Highly Educated Iranian Refugees from Sweden. Journal of International Migration and Integration, 17 (3), pp. 649-667.

Kohlbacher J. and Reeger U. (2020), Globalization, Immigration and Ethnic Diversity: The Exceptional Case of Vienna. In: Musterd S. (ed.), Handbook on Urban Segregation, pp. 101-117. London, Edward Elgar Publishing Ltd.

KonRad Adenauer Foundation (2016), The Role of Cities Regarding the Integration of Refugees: Chances and Perspectives $<\mathrm{http}: / /$ www.kas.de/hamburg/de/publications/44285/> [accessed 22-01-2020].

Kordel S., Weidinger T. und HaChMeISTER S. (2018), Lebenswelten geflüchteter Menschen in ländlichen Regionen qualitativ erforschen. Methodische Überlegungen zu einem partizipativ orientierten Forschungsansatz Thünen Working Paper $106<$ https://www.gefluechtete-inlaendlichen-raeumen.de/fileadmin/gilr/pdfs/Thuenen_Working_Paper_106.pdf> [Zugriff 19-01-2020].

Lindley A. and VAN Hear N. (2007), New Europeans on the move: a preliminary review of the onward migration of refugees within the European Union. (Centre on Migration, Policy and Society, Working paper, 57).

LUNDGREN S. (2018), Migration patterns of refugee immigrants. Evidence from Swedish municipalities MA Thesis, Economics, Jönköping University <http://www.diva-portal.org/ smash/get/diva2:1218126/FULLTEXT01.pdf> [accessed 07-01-2020].

Malmberg B., Nielsen M.M., Andersson E. and HaAndrikman K. (2016), Residential Segregation of European and Non-European Migrants in Sweden: 1990-2012 (ResSegr Working Paper No. 2016, 1).

Manea E. et al. (2017), Parallelgesellschaften. Segregation und desintegrative Milieus, Perspektiven Integration 06/2017, ÖIF (ed.). <https://www.integrationsfonds.at/fileadmin/content/ AT/Fotos/Logos_Sujets/Allgemeine/3_Perspektiven_Parallelgesellschaft_200x260_ SCREEN.pdf $>$ [Zugriff 27-01-2020].

Marcińczak S., Tammaru T., Novák J., Gentile M., Kovács Z., Temelová J., Valatka V., KäHRIK A. and SzaBó B. (2015), Patterns of socio-economic segregation in the capital cities of fast-track reforming postsocialist countries. Annals of the American Association of Geographers, 105(1), pp.183-202.

McAuliffe M. and JAYASURIYA D. (2016), Do asylum seekers and refugees choose destination countries? Evidence from large-scale surveys in Australia, Afghanistan, Bangladesh, Pakistan and Sri Lanka, International Migration, 54 (4), pp. 44-59 <https:// doi.org/10.1111/ imig. 12240> [accessed 14-01-2020].

MüLLER W. (11-08-2016), Mindestsicherung zieht Asylberechtigte verstärkt nach Wien, Der Standard<https://derstandard.at/2000042670101/Mindestsicherung-zieht-Asylberechtigteverstaerkt-nach-Wien> [Zugriff 14-08-2019].

Musterd S. (2020), Handbook on Urban Segregation. London, Edward Elgar Publishing Ltd.

Musterd S. and Kempen van R. (2000), The spatial dimensions of urban social exclusion and integration: A European comparison. Amsterdam, Universiteit van Amsterdam.

ORF (04-05-2019), 15 Flüchtlingsheime vor der Schließung <https://ooe.orf.at/v2/news/stories/ 2979541/> [Zugriff 22-01-2020]. 
Phillips D. (2006), Moving towards integration: the housing of asylum seekers and refugees in Britain, Housing Studies, 21, pp. 539-53.

Robinson V. and Segrott J. (2002), Understanding the Decision-Making of Asylum Seekers. Home Office Research Study, 243. London, Home Office Research, Development and Statistics Directorate, $<\mathrm{http} / /$ citeseerx.ist.psu.edu/viewdoc/download?doi $=10.1 .1 .473 .3461 \& \mathrm{rep}=$ rep1\&type $=$ pdf $>$ [accessed 10-12-2019].

Robinson V., ANDERSSON R. and Musterd S. (2003), Spreading the 'burden': A review of policies to disperse asylum seekers and refugees. Bristol, Bristol University Press; Policy Press.

RogelJ B. (2017), The Changing Spatiality of the "European Refugee/Migrant Crisis", MET (Migracijske i etnicke teme), 33 (2), pp. 191-219 <https://doi.org/10.11567/met.33.2.4> [accessed 02-11-2019].

Sabates-Wheeler R. and Waite M. (2003), Migration and Social Protection: A Concept Paper (Development Research Centre on Migration, Globalisation and Poverty Working Paper Series, T2). Sussex, Sussex University <https://www.researchgate.net/profile/Rachel_ Sabates-Wheeler/publication/242553905_Migration_and_Social_Protection_A_Concept_ Paper/links/02e7e538867037fa36000000.pdf> [Zugriff 28-01-2020].

Stadt Wien, Magistratsabteilung 17 (ed.) (o.J.), Leitlinien der Wiener Integrations- und Diversitätspolitik. <https://www.wien.gv.at/menschen/integration/pdf/integrationsleitli nien.pdf $>$ [Zugriff 29-11-2019].

SzIGETVARI A. (22-01-2020), ÖVP: Arbeitslose Asylberechtigte im Osten sollen Jobs im Westen annehmen, Der Standard <https://www.derstandard.at/story/2000113624204/oevp-willjobvermittlung-von-asylberechtigten-forcieren $>$ [Zugriff 22-01-2020].

Thielemann E., Williams R., Boswell C. and Matrix Insight Ltd. (2010), What system of burden-sharing between Member States for the reception of asylum seekers? Study. Directorate General for Internal Policies, Policy Department C: Citizens‘ Rights and Constitutional Affairs, Civil Liberties, Justice and Home Affairs, European Parliament, Brussels.

Wiener Zeitung (14-03-2016), Proteste nicht nur gegen Asylquartier, Wiener Zeitung, <https:// www.wienerzeitung.at/nachrichten/politik/wien/806405-Proteste-nicht-nur-gegenAsylquartier.html $>$ [Zugriff 21-01-2020].

Winroither E. und Thalhammer A. (04-06-2017), Mindestsicherung. Das große Wandern: Wer zieht des Geldes wegen nach Wien? Die Presse <https://diepresse.com/home/panorama/ wien/5229335/Mindestsicherung_Wer-zieht-des-Geldes-wegen-nach-Wien> [Zugriff 1211-2019].

ZaVodny M., (1999), Determinants of recent immigrants' locational choices. International Migration Review, 33 (4), pp. 1014-1030.

Zorlu A. and Mulder C.H. (2008), Initial and Subsequent Location Choices of Immigrants to the Netherlands. Regional Studies, 42 (2), pp. 245-264. 